\title{
Cardiorespiratory fitness and mnemonic discrimination across the adult lifespan
}

\author{
Rachel K. Nauer, ${ }^{1,2,3,4}$ Karin Schon, ${ }^{1,2,3,4,5}$ and Chantal E. Stern ${ }^{1,2,3,5}$ \\ ${ }^{1}$ Department of Psychological and Brain Sciences, Boston University, Boston, Massachusetts 02215, USA; ${ }^{2}$ Center for Memory and \\ Brain, Boston University, Boston, Massachusetts 02215, USA; ${ }^{3}$ Center for Systems Neuroscience, Boston University, Boston, \\ Massachusetts 02215, USA; ${ }^{4}$ Department of Anatomy and Neurobiology, Boston University School of Medicine, Boston, \\ Massachusetts 02118, USA
}

\begin{abstract}
With a rising aging population, it is important to develop behavioral tasks that assess and track cognitive decline, and to identify protective factors that promote healthy brain aging. Mnemonic discrimination tasks that rely on pattern separation mechanisms are a promising metric to detect subtle age-related memory impairments. Behavioral performance on these tasks rely on the integrity of the hippocampus and surrounding circuitry, which are brain regions known to be adversely affected in aging and neurodegenerative disorders. Aerobic exercise, which improves cardiorespiratory fitness (CRF), has been shown to counteract aging-related decreases in structural and functional brain integrity and attenuate decline of cognitive performance. Here, we tested the hypothesis that higher CRF attenuates age-related deficits in mnemonic discrimination in both a nonspatial mnemonic discrimination (Mnemonic Similarity Task) and a virtual navigation task (Route Disambiguation Task). Importantly, we included individuals across the lifespan (aged 18-83 yr), including the middle-age range, to determine mnemonic discrimination performance across adulthood. Participants completed two mnemonic discrimination tasks and a treadmill test to assess CRF. Our results demonstrate robust negative age-related effects on mnemonic discrimination performance across both the nonspatial and spatial domains. Critically, higher CRF mitigated age-related attenuation in spatial contextual discrimination task performance, but did not show an attenuation effect on performance for object-based mnemonic discrimination. These results suggest that performance on spatial mnemonic discrimination may be a useful tool to track vulnerability in older individuals at risk for cognitive decline, and that higher CRF may lead to cognitive preservation across the adult lifespan, particularly for spatial disambiguation of similar contexts.
\end{abstract}

[Supplemental material is available for this article.]

Physical fitness and aerobic exercise have emerged as effective protective factors to promote healthy brain aging (Barnes et al. 2003; Burns et al. 2008; Erickson et al. 2011; Lautenschlager et al. 2012; Brown et al. 2013) and attenuate both cognitive decline (Barnes et al. 2003; Brown et al. 2013) and brain atrophy (Burns et al. 2008; Brown et al. 2013). While recent studies have examined the impact of aerobic exercise on brain health, the parameters of the relationship between cardiorespiratory fitness (CRF) and cognition, including how this relationship may change across the adult lifespan, remains unclear. Inclusion of individuals across the adult lifespan is critical to assess if middle-age may be the start of subtle cognitive and structural change that is predictive of future neuropathological impairment (Clark et al. 2016; Melah et al. 2016).

The hippocampus and entorhinal cortex are essential brain regions for encoding, consolidating and retrieving episodic events. This circuitry is responsible for orthogonalizing similar incoming information into distinct neural representations to avoid interference during memory formation, a process known as pattern separation (Marr 1971). Along with contributions to pattern separation, the entorhinal-hippocampal circuitry is critical for tasks of allocentric, or world-centered, navigation in both rodents (Hafting et al. 2005; McNaughton et al. 2006; Killian et al. 2012) and humans (Doeller et al. 2010; Jacobs et al. 2013; Brown and Stern 2014; Brown et al. 2014). Spatial disambiguation of highly similar

${ }^{5}$ Denotes co-senior authorship.

Corresponding author: rnauer@bu.edu

Article is online at http://www.learnmem.org/cgi/doi/10.1101//m.049197.118. contexts, likely achieved at least partially via pattern separation mechanisms, is critical for the accurate formation and subsequent retrieval of spatial memories. In healthy aging, a loss of entorhinalhippocampal communication (Raz et al. 2004; Yassa et al. 2010a) is associated with decreased cognitive functioning on hippocampally dependent memory tasks (Yassa et al. 2010a). This, in turn, results in aging-related impairments on behavioral performance of medial temporal lobe-dependent spatial (Moffat et al. 2006; Stark et al. 2010; Holden and Gilbert 2012; Reagh et al. 2014; Williams et al. 2019) and nonspatial (Stark et al. 2010, 2013; Yassa et al. 2011; Ly et al. 2013) memory tasks that likely require pattern separation.

Corroborating evidence from rodent studies demonstrate an aging-related reduction in plasticity within the local entorhinalhippocampal circuitry (Geinisman et al. 1992; Kuhn et al. 1996), resulting in lower mnemonic flexibility and poorer performance on cognitive tasks of mnemonic discrimination (Creer et al. 2010; Sahay et al. 2011). Physical exercise seems to reverse many of the aging-related effects on plasticity in rodents (Nokia et al. 2016) and acts to promote angiogenesis and synaptogenesis across the brain as well as adult hippocampal neurogenesis (AHN) (van Praag et al. 1999, 2005). This suggests exercise interventions can

2020 Nauer et al. This article is distributed exclusively by Cold Spring Harbor Laboratory Press for the first 12 months after the full-issue publication date (see http://learnmem.cshlp.org/site/misc/terms.xhtml). After 12 months, it is available under a Creative Commons License (AttributionNonCommercial 4.0 International), as described at http://creativecommons. org/licenses/by-nc/4.0/. 
promote healthy cognitive aging by mitigating cognitive decline and promoting better performance on tasks that rely on pattern separation mechanisms (Creer et al. 2010; Vivar et al. 2016).

Clinical exercise trials and correlational studies in older adult humans show a link between higher CRF and increased hippocampal volume (Erickson et al. 2009, 2011; Pereira et al. 2007; Honea et al. 2009) and spatial working memory (Erickson et al. 2011). In healthy young adults, both aerobic exercise training (Déry et al. 2013) and an acute bout of exercise (Suwabe et al. 2017b, 2018) have been linked to better performance on mnemonic discrimination tasks, but how CRF relates to performance on these tasks in middle-aged and older populations is less clear. Importantly, middle-age may be the ideal time to implement neuroprotective interventions such as aerobic exercise (Tolppanen et al. 2014). In a study by Holzschneider et al. (2012), the authors demonstrated a positive association between CRF and hippocampal activation during spatial maze learning in adults between the ages of 40-55 yr using fMRI, suggesting aerobic exercise may enhance spatial memory and virtual navigation in a middle-aged population. Conceivably, increasing CRF through aerobic exercise has the potential to modulate functional and structural plasticity in the hippocampus throughout the lifespan, facilitating the formation and retention of memories with overlapping (OL) features in both the spatial and nonspatial domains.

In the current study, healthy adults between the ages of $18-83 \mathrm{yr}(\mathrm{N}=80)$ performed two hippocampally dependent mne- monic discrimination tasks, completed a neuropsychological test battery, and underwent a treadmill test to assess CRF. The Mnemonic Similarity Task (MST; Fig. 1B) is an established objectbased task known to reliably assess mnemonic discrimination in humans. Performance on this task is thought to depend, at least in part, on pattern separation and has been shown to be sensitive to aging (Yassa et al. 2011; Stark et al. 2013; Stark and Stark 2017), structural integrity of the hippocampus (Kirwan et al. 2012), and disease status (Yassa et al. 2010b). This task has been shown to elicit pattern-separation like activity in the DG/CA3 in fMRI studies (Kirwan et al. 2007; Bakker et al. 2008). Additionally, participants performed a Route Disambiguation Task (RDT, Fig. 1C) that involves encoding and retrieval of OL spatial sequences previously used with young adults (Brown et al. 2010, 2012, 2014). Previous fMRI experiments using this task show evidence for DG/CA3 recruitment, particularly in conditions when subjects have to make navigational choices during an OL portion of two mazes (Brown et al. 2010, 2012, 2014). In this paradigm, successful navigation during task performance is dependent on accurate spatial memory that relies, in part, on disambiguating mazes that share OL hallways (i.e., spatial pattern separation). Using these two tasks and a treadmill-based assessment of CRF, we tested the predictions that (i) there is a negative relationship between increased age and mnemonic flexibility in both the nonspatial and spatial domains, and (ii) greater CRF attenuates this negative relationship.

A
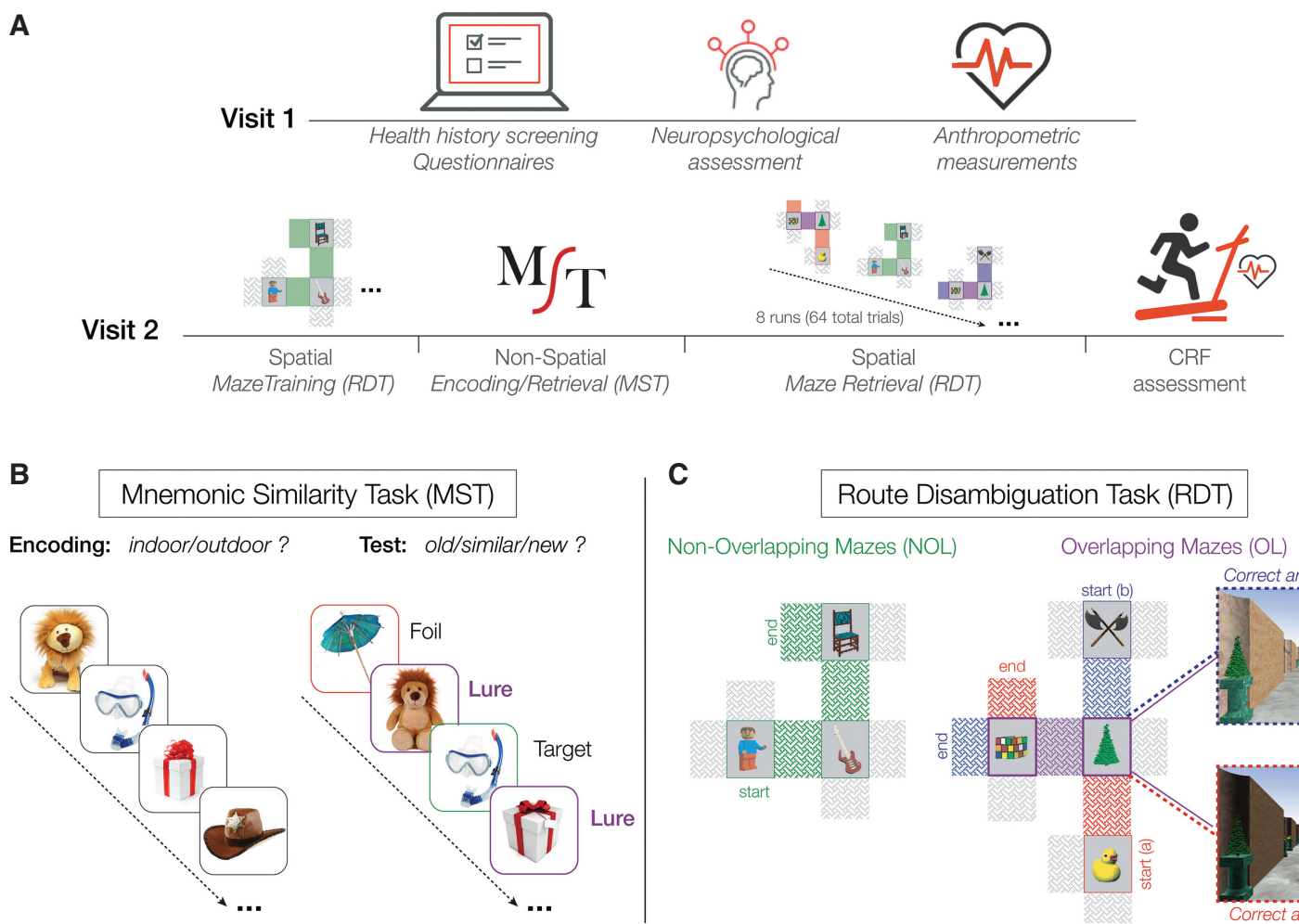

Route Disambiguation Task (RDT)

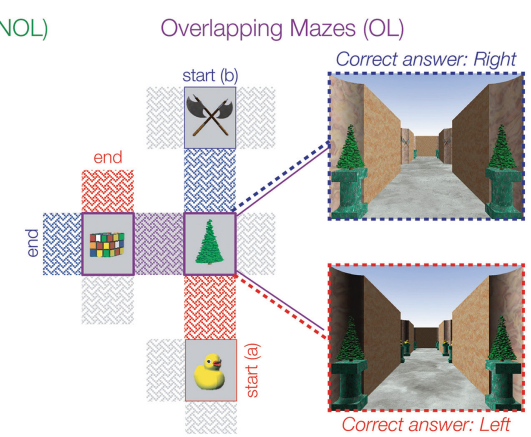

Figure 1. Overview of experimental procedures. $(A)$ Outline of study timeline. Participants came to the laboratory for two study visits. (B) Mnemonic Similarity Task (MST) design consisted of an incidental encoding phase (left) and a surprise recognition memory test (right). The recognition memory test contained three stimulus conditions: exact repetitions of objects shown during encoding (Targets; outlined in green), similar objects but not exactly identical (Lures; outlined in purple), and novel objects (Foils; outlined in red). Participants responded to the objects with old/similar/new classification. (C) Route disambiguation task (RDT) design. An example of a nonoverlapping (NOL) maze trajectory is shown on the left in green and an example $\mathrm{OL}$ maze is shown on the right. Start and end locations are shown for each example maze. NOL mazes were distinct from each other, and the response at each intersection remained consistent. For OL mazes, start and end locations were distinct and shown in blue and red colors for illustration. The critical OL choice points and hallway are colored in purple. Insets for critical choice point 2 show examples of the perspective at this intersection and demonstrate how the correct answer is dependent on which maze location the participant started in. 


\section{Results}

\section{Participant demographics}

Participant age groups were as follows: $18-30$ yr of age (Young Adults $(\mathrm{YA}) ; \mathrm{N}=17$, mean age $=23.12 \pm 3.1 \mathrm{yr}, 8$ female), 31-49 yr of age (Middle-Younger adults (MY); $\mathrm{N}=23$, mean age $=38.96 \pm 6.1 \mathrm{yr}, 10$ female), 50-66 yr of age (Middle-Older $(\mathrm{MO}) ; \mathrm{N}=21$, mean age $=$ $59.81 \pm 5.3 \mathrm{yr}, 11 \mathrm{female}$ ), and $67-83 \mathrm{yr}$ of age (Older Adults (OA); $\mathrm{N}=19$, mean age $=73.58 \pm 5.1 \mathrm{yr}, 12$ female). Participant demographic information, CRF data, anthropometric measures and neuropsychological assessment data are summarized in Table 1. Additional assessments of neuropsychological data are described in Supplemental Information. Participant performance on these neuropsychological measures fell within the range for normative data (Strauss et al., 2006). Correlations between Rey-Auditory Verbal Learning Test (RAVLT) performance and spatial and nonspatial task performance are described in a subsequent section.

\section{Experiment 1}

\section{Age effects on nonspatial mnemonic discrimination}

In Experiment 1, we sought to first replicate previous work demonstrating age-related effects on mnemonic discrimination using the MST (Stark et al. 2013, 2015), and then determine whether CRF attenuates this effect. First, we calculated the Lure Discrimination Index (LDI) by taking the difference between the rate of "similar" responses to lure stimuli and the rate of "similar" responses to foils. We also calculated a more traditional score of recognition memory (REC), operationalized as the difference between the rate of a response of "old" to target stimuli and the rate of "old" responses to foil stimuli. These scores allow us to correct for any response bias on a per-subject basis. Replicating previous work using the same task and similar statistical analyses (Stark et al. 2013), we found a significant negative relationship between age and LDI $(r$ $=-0.55, t_{(76)}=-5.78, P<0.001$; regression model $F_{(3,74)}=11.4$, adjusted $R^{2}=0.29, P<0.001$; Fig. $\left.2 \mathrm{~A}\right)$, but no relationship between age and REC $\left(r=-0.02, t_{(76)}=-0.18, P=0.86\right.$; regression model $F_{(3,74)}=1.46$, adjusted $\left.R^{2}=0.02, P>0.05\right)$, indicating age is associat- ed with mnemonic discrimination but not overall recognition memory. Next, we separated the subjects into the four age groups. Using one-way analysis of variance (ANOVA), we demonstrated a significant main effect of age group on $\operatorname{LDI}\left(F_{(3,72)}=7.63\right.$, $P<0.001, \eta^{2}=0.237$; Fig. $\left.2 \mathrm{~B}\right)$ but not $\operatorname{REC}\left(F_{(3,72)}=1.62, P>0.1\right.$, $\eta^{2}=0.06$; Fig. 2C). Tukey HSD post-hoc testing revealed that the LDI score was significantly higher in the youngest group (YA $\mathrm{LDI}_{\text {mean }}=0.41 \pm 0.16$ ) compared to the two oldest two groups $\left(\mathrm{MO} \mathrm{LDI}\right.$ mean $=0.14 \pm 0.26, \mathrm{OA} \mathrm{LDI}_{\text {mean }}=0.11 \pm 0.16$; all $\mathrm{Ps}<0.05$, Fig. 2B).

We next performed analyses on response distribution (i.e., rates of calling a target object "old," "similar," or "new"). The response distributions are shown in Figure 3A. Consistent with previous reports using this task (Stark et al. 2013), correct categorization of targets as "old" and foils as "new" were high and stable across all age groups. To assess this statistically, we examined the effect of age group on response proportion for $\mathrm{p}$ ("old"|target) and the $\mathrm{p}$ ("new"|foil) using separate one-way ANOVAs. As predicted, there was no effect of age group on correct categorization for responding "old" for the target condition $\left(F_{(3,73)}=1.52, P=0.22, \eta^{2}=\right.$ 0.02 ; Fig. 3A left panel, “ $\mathrm{O}$ "|Target across age groups), nor was there an effect of age group on correctly responding "new" for the foil condition $\left(F_{(3,73)}=1.24, P=0.30, \eta^{2}=0.01\right.$; Fig. 3A right panel, "N"|Foil across age groups). We were primarily interested in the proportion of responses of correctly identifying lures as "similar." Results of this one-way ANOVA demonstrated a significant effect of age group $\left(F_{(3,73)}=9.58, P<0.001, \eta^{2}=0.25\right)$. Tukey HSD posthoc testing revealed that older age had a negative effect on correctly identifying lures as "similar." Specifically, although the YA cohort was significantly better at this identification than the MO and OA group, they did not differ from the MY group. The MY group was significantly better than the OA group, but did not differ from the MO group. The two oldest groups did not differ from each other. Analysis of the age effects on responses of incorrectly identifying lures as "old" revealed a complementary age-related effect showing that older adults misidentified lures as "old" $\left(F_{(3,73)}=\right.$ 7.81, $P<0.001, \eta^{2}=0.21$; Fig. 3A, middle panel, "S"|Lure). Together, these results show that the response distribution for the lure condition differed as a function of age. The YA cohort demonstrated

Table 1. Participant characteristics

\begin{tabular}{|c|c|c|c|c|c|}
\hline & $\mathbf{N}$ & Group 1 Ages $18-30$ & Group 2 Ages 31-49 & Group 3 Ages 50-66 & Group 4 Ages 67-85 \\
\hline \multicolumn{6}{|l|}{ Demographics } \\
\hline $\mathrm{N}$ & 80 & 17 & 23 & 21 & 19 \\
\hline Age (years) & & $23.35(3.22)$ & $39.0(6.1)$ & $59.81(5.26)$ & $73.68(4.96)$ \\
\hline Education (years) & & $15.65(1.80)$ & $16.52(2.57)$ & $17.28(2.28)$ & $16.84(2.24)$ \\
\hline Sex & & $9 \mathrm{M}, 8 \mathrm{~F}$ & $13 \mathrm{M}, 10 \mathrm{~F}$ & $10 \mathrm{M}, 11 \mathrm{~F}$ & $7 \mathrm{M}, 12 \mathrm{~F}$ \\
\hline \multicolumn{6}{|l|}{ Physiology } \\
\hline $\mathrm{VO}_{2 \operatorname{MAX}}\left(\mathrm{L} \mathrm{min}^{-1}\right)^{\mathrm{a}}$ & 62 & $45.12(7.95)$ & $36.36(6.02)$ & $39.08(7.74)$ & $31.42(5.86)$ \\
\hline $\operatorname{BMI}\left(\mathrm{kg} \cdot \mathrm{m}^{-2}\right)^{\mathrm{a}}$ & 80 & $23.54(3.24)$ & $27.38(6.23)$ & $25.6(5.25)$ & $26.72(3.69)$ \\
\hline Seated resting HR & 80 & $68.29(6.84)$ & 73.96 (10.32) & $63.90(10.72)$ & $67.63(13.96)$ \\
\hline Waist/Hip Ratio ${ }^{a}$ & 80 & $0.79(0.088)$ & $0.83(0.08)^{*}$ & $0.85(0.11)$ & $0.87(0.08)$ \\
\hline \multicolumn{6}{|l|}{ Neuropsych } \\
\hline SBSOD & 80 & $4.87(0.89)$ & $4.82(1.22)$ & $5.17(0.87)$ & $4.99(0.75)$ \\
\hline RAVLT Total Score ${ }^{a}$ & 80 & $58.63(7.62)$ & $58.09(9.86)$ & $52.57(8.41)^{\star}$ & $46.63(8.55)^{\star}$ \\
\hline RAVLT Immediate ${ }^{\mathrm{a}}$ & 80 & $12.53(2.94)$ & $12.65(2.64)$ & $10.38(3.47)^{*}$ & $9.42(2.71)$ \\
\hline RAVLT Long Delay ${ }^{\mathrm{a}}$ & 80 & $12.88(2.47)$ & $12.43(2.68)$ & $10.62(3.71)^{*}$ & $8.32(3.30)$ \\
\hline TMT B/A Ratio & 80 & $2.70(2.15)$ & $2.22(0.63)$ & $2.22(0.64)$ & $2.43(0.79)$ \\
\hline VST Ratio ${ }^{a}$ & 80 & $1.61(0.27)$ & $1.64(0.38)$ & $1.94(0.39)$ & $2.11(0.54)$ \\
\hline DRS- $2_{\text {raw }}{ }^{b}$ & 36 & - & - & $141.94(2.01)$ & $140.95(2.17)$ \\
\hline
\end{tabular}

All data are reported as mean (sd).

BMI, Body Mass Index; HR, Heart Rate (beats per minute); SBSOD, Santa Barbara Sense of Direction; RAVLT, Rey-Auditory Verbal Learning Test; TMT, Trail Making Test; VST, Victoria Stroop Test; DRS, Dementia Rating Scale

*Significant $(P<0.05)$ sex differences within age group.

${ }^{a}$ Main effect of age in a multiple regression adjusting for sex and education.

${ }^{\mathrm{b}}$ Only subjects aged 55+ completed the DRS-2. 

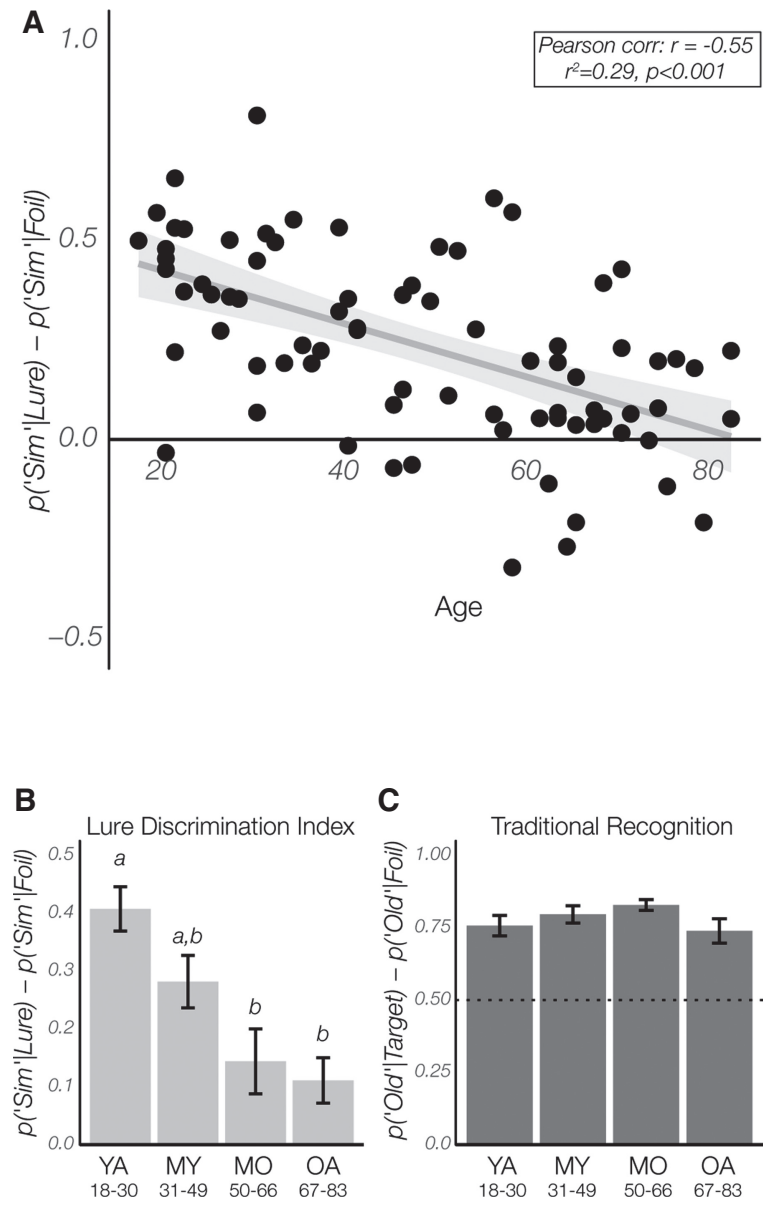

Figure 2. Age effects on nonspatial pattern separation in the Mnemonic Similarity Task (MST). (A) LDI score across the adult lifespan. Strong linear decrease in lure discrimination (LDI) performance, $(B) \mathrm{LDI}$ by age group. The young adult group (YA, ages 18-30) shows highest performance, whereas mnemonic discrimination in the oldest two groups (middle-older; $\mathrm{MO}$, ages 50-66 and older adults; OA, ages 67-83) is significantly diminished compared to the YA group. Groups sharing letters ( $a$ or $b$ ) signify no significant difference for LDI. Groups that do not share the same letter signify a significant difference in LDI score. (C) Traditional recognition by age group. In contrast to the LDI results, age related effects are not present on recognition performance.

a higher response proportion for correctly identifying lure trials as "similar" than "old" (Fig. 3A, top middle panel). This effect changed in the opposite direction with increasing age. For the MY age group, lure trial response proportion was more equally identified as "similar" and "old." Response distributions for lure trials in the two oldest cohorts (MO and OA) show a switch, such that they are misidentifying lure trials as "old" significantly more often than correctly identifying lure trials as similar (Fig. 3A). It is of note that all age-related analyses reported to this point included all subjects that completed the MST $(\mathrm{N}=78)$, however, when we repeated the analyses using only the subset of subjects who successfully completed both the MST and CRF test $(\mathrm{N}=60)$, the results remain unchanged. The average response proportions for the MST by age group are listed in Supplemental Information, Table S1.

\section{Interactions between age and CRF on nonspatial mnemonic discrimination}

Next, we set out to elucidate how CRF might predict mnemonic discrimination. All analyses are limited to only those participants who successfully completed both the fitness assessment and MST $(\mathrm{N}=60)$. To elucidate the potential differential effects of CRF across the adult lifespan, all analyses included age and CRF as main effects, as well as an interaction term of age by CRF. Results of a multiple regression $\left(F_{(5,54)}=4.3, P<0.01\right)$ showed a significant main effect of age $\left(F_{(1,54)}=19.96, P<0.001\right)$, but no main effect of estimated $\dot{\mathrm{V}} \mathrm{O}_{2 \mathrm{MAX}}\left(F_{(1,54)}=0.008, P>0.05\right)$, nor an interaction of age by CRF $\left(F_{(1,54)}=.29, P>0.05\right)$ on LDI. This lack of main effect of estimated $\dot{\mathrm{VO}}_{2 \mathrm{MAX}}$ and interaction is likely due to the known high collinearity between age and estimated $\dot{\mathrm{V}}_{2 \mathrm{MAX}}$, our measure of CRF (ACSM 2014). Additionally, on average, women have lower $\dot{\mathrm{V}} \mathrm{O}_{2 \mathrm{MAX}}$ values than age-matched men (ACSM 2014). To partially account for these issues, rather than using estimated $\dot{\mathrm{VO}}_{2 \mathrm{MAX}}$ as a continuous variable, we split the participants into higher-fit or lower-fit groups based on the median $\dot{\mathrm{VO}}_{2 \mathrm{MAX}}$ for our sample by sex within their age bin. For example, for women in the YA group, the median estimated $\dot{\mathrm{VO}}_{2 \mathrm{MAX}}$ value was $33.97\left(\mathrm{~mL} \mathrm{O}_{2} /(\mathrm{kg}\right.$ min) whereas for men in the same YA age group, the median estimated $\dot{\mathrm{V}} \mathrm{O}_{2 \mathrm{MAX}}$ value was $51.94\left(\mathrm{~mL} \mathrm{O}_{2} /(\mathrm{kg}\right.$ min; see Supplemental Fig. 1). We entered the data into a $4 \times 2$ ANOVA to determine the main and interactive effects of age group (YA, MY, MO, OA) and CRF level (higher-fit, lower-fit) on LDI. Results from this analysis recapture the significant main effect of age $\left(F_{(3,50)}=5.376, P<0.01, \eta^{2}=\right.$ 0.24 ), but show no main effect of CRF level (higher vs. lower; $\left.F_{(1,50)}=0.24, P>0.05, \eta^{2}=0.004\right)$ nor interaction effect between age group by CRF level $\left(F_{(3,50)}=0.3, P>0.05, \eta^{2}=0.013\right)$. As expected for our measure of traditional recognition (REC), we found no main effects of age group $\left(F_{(3,50)}=1.38, P>0.05, \eta^{2}=.07\right)$ or CRF level $\left(F_{(1,50)}=1.96, P>0.05, \eta^{2}=0.03\right)$, nor was there an interaction between age group by CRF level $\left(F_{(3,50)}=0.06, P>0.05, \eta^{2}=0.004\right)$. Average performance for each age group and CRF level are in Supplemental Information, Table S2.

Next, we ran follow-up analyses to determine if there were effects of CRF level on the proportion of correct responses for the lures, given the previous results showing age specific effects on lures only. We ran a $4 \times 2$ ANOVA to predict the accurate response of "similar" for lure trials as predicted by age group (YA, MY, MO, $\mathrm{OA}$ ) and CRF level (higher-fit, lower-fit), including an interaction term between the predictors. This analysis demonstrated a significant effect of age group $\left(F_{(3,51)}=5.91, P=0.002, \eta^{2}=0.2\right)$, but no effect of CRF level $\left(F_{(1,51)}=0.11, \quad P=0.75, \quad \eta^{2}=0.02\right)$, nor an interaction effect between age group and CRF level $\left(F_{(3,51)}=1.07\right.$, $P=0.37, \eta^{2}=0.001$ ). Together, these results suggest that performance on the MST does not seem to be sensitive to CRF level across any age group.

\section{Experiment 2}

\section{Age effects on spatial disambiguation}

Prior to examining the potential effects of age and CRF on accuracy in the RDT, we sought to examine the main and interactive effects of condition (OL, NOL) and choice point (CP; CP1, CP2, CP3). We predicted that accuracy on the NOL mazes would be higher than accuracy on the OL mazes, and this effect would be specific for choice point 2 and choice point 3 , given that accurate responses at these choice points are context-dependent, thus theoretically requiring disambiguation effort or spatial pattern separation. Using a linear mixed-effects model, we specified condition (OL, NOL) and choice point (CP1, CP2, CP3) as fixed-effects, sex and education as covariates and subject as a random effect. We found significant main effects of condition $\left(F_{(1,395)}=43.27, P<0.001\right)$ and choice point $\left(F_{(2,395)}=12.94, P<0.001\right)$, as well as a significant interaction between condition and choice point $\left(F_{(2,395)}=31.88, P<0.001\right)$. Tukey post-hoc comparisons from the model confirmed that overall, accuracy on mazes for the nonambiguous choice point 1 did 
A
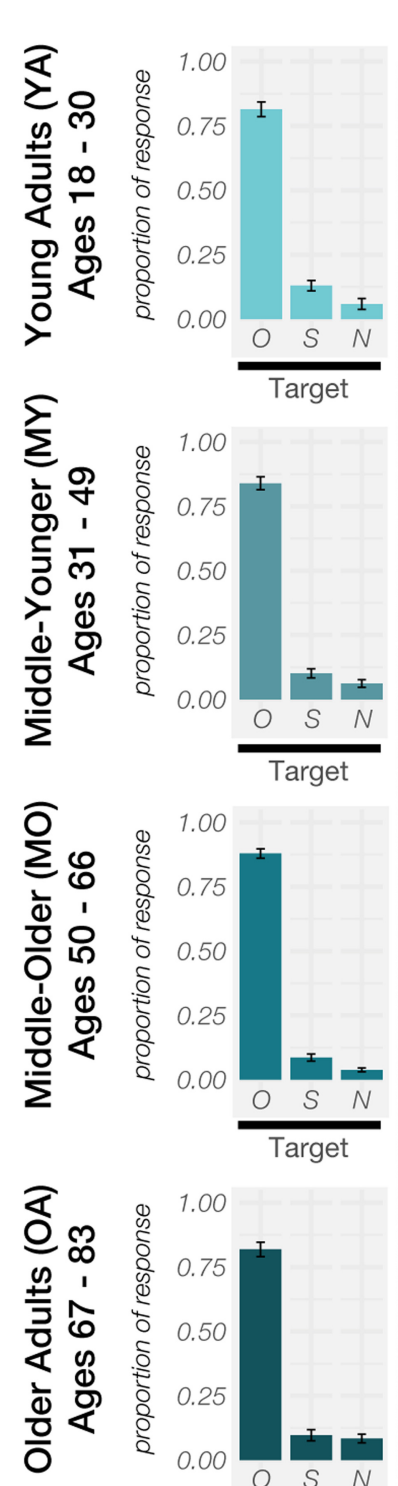
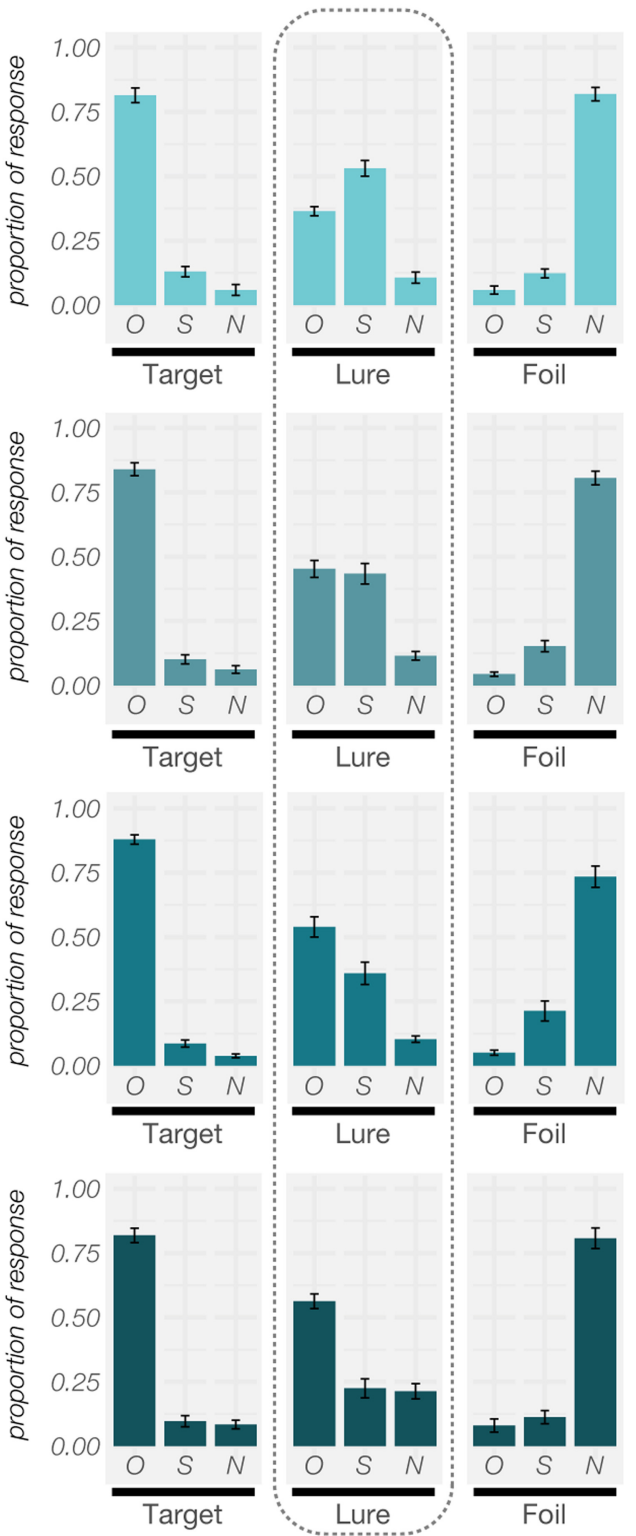

B
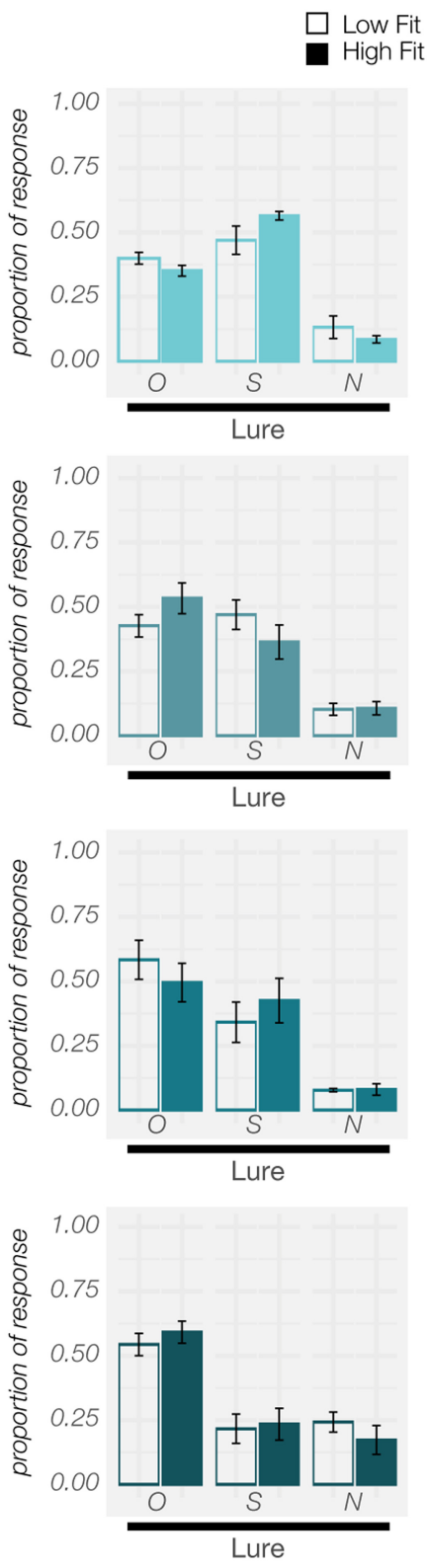

Figure 3. Characterization of response proportion in the MST. (A) Mean response proportion (old, similar, new) for each of the three conditions (target, lure, foil) is displayed by age group from youngest (YA, top) to oldest cohort (OA, bottom). Dotted circle highlights the lure trials, for which response proportion changes as a function of age. YA (top) correctly identify lure trials as similar more than incorrectly identify them as old. This gradually interchanges as a function of age, with the middle-younger (MY) group responding to lures as "old" and "similar" equivocally and the older two groups (middle-older; $\mathrm{MO}$ and $\mathrm{OA}$ ) mischaracterizing lures as "old" disproportionately more "similar". (B) CRF level does not significantly affect response proportion across any age group.

not differ between OL and NOL conditions $\left(\mathrm{CP} 1_{\mathrm{NOL}}\right.$ mean $0.64 \pm$ $\left.0.21 ; \mathrm{CP} 1_{\mathrm{OL} \text { mean }} 0.69 \pm 0.21 ; P>0.05\right)$. However, at critical intersections where participants required disambiguation $\left(\mathrm{CP} 2_{\mathrm{OL}}\right.$ mean $0.53 \pm 0.19 ; \mathrm{CP} 3_{\mathrm{OL} \text { mean }} 0.57 \pm 0.18$ ), participants were significantly less accurate than at the corresponding non-OL intersections $\left(\mathrm{CP} 2_{\mathrm{NOL} \text { mean }} 0.67 \pm 0.22 ; \mathrm{CP} 3_{\mathrm{NOL} \text { mean }} 0.71 \pm 0.22 ; \mathrm{Ps}<0.05\right.$, Fig. $\left.4 \mathrm{~A}\right)$.

To investigate the effect of age on maze accuracy, we first performed Pearson correlations between age and overall maze accuracy and separately for NOL and OL maze accuracy. As expected, we found significant negative correlations between age and overall maze accuracy $\left(r=-0.59, t_{(78)}=-6.41, P<0.001\right)$, age and NOL maze accuracy $\left(r=-0.55, t_{(78)}=-5.76, P<0.001\right)$, and age and OL maze accuracy $\left(r=-0.58, t_{(78)}=-6.32, P<0.001\right)$. Next, we separated the participants into age groups and entered the data into a rm-ANOVA with condition (OL, NOL) and choice point (CP1, $\mathrm{CP} 2, \mathrm{CP} 3$ ) as within-subject factors, and age group (YA, MY, MO, $\mathrm{OA}$ ) as a between-subject factor. The model also included a threeway interaction term for condition by choice point by age group, with the specific hypothesis that there would be a negative effect of age group, which would be greater for OL mazes compared to NOL mazes at choice point 2 and choice point 3 . Results showed main effects of condition $\left(F_{(1,453)}=20.88, P<0.001, \eta^{2}=0.03\right)$, age group $\left(F_{(3,453)}=39.71, \quad P<0.001 ; \eta^{2}=0.16\right)$, and choice point $\left(F_{(2,453)}=6.25, P<0.01, \eta^{2}=0.02\right)$. As predicted, the three-way 
A

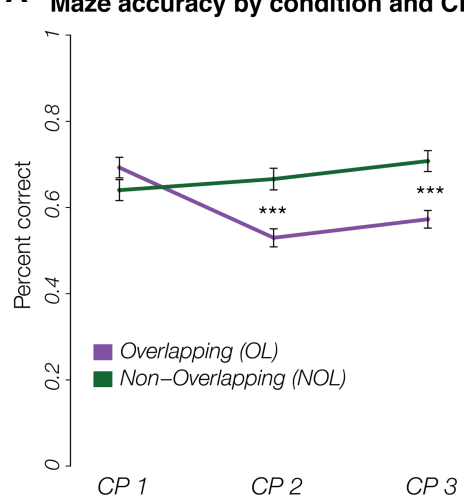

B

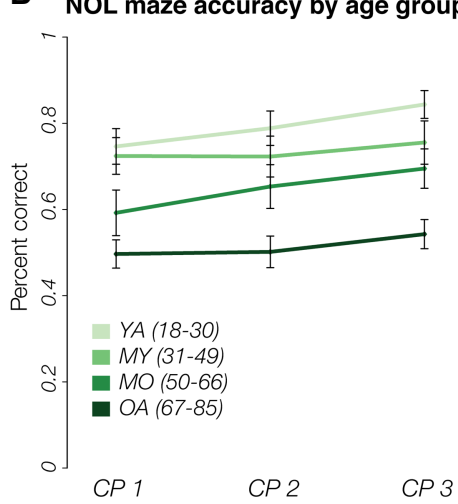

C OL maze accuracy by age group

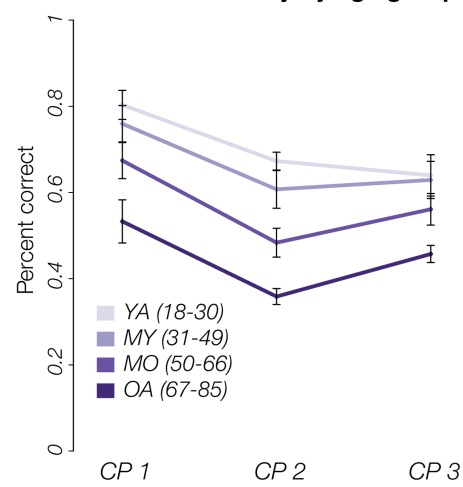

Figure 4. Performance on the route disambiguation task (RDT). (A) Performance was significantly lower for critical OL choice points (CP) 2 and $3\left({ }^{* * *}\right) P<$ 0.001. Comparing across the four age groups, there is a robust age-effect for accuracy in NOL mazes $(B)$ and OL mazes (C). The age-effect is particularly pronounced in critical OL maze CPs 2 and 3, when additional contextual disambiguation was required for successful navigation. Age groups were as follows: Young Adult (YA; ages 18-30, N=17); Middle-Younger (MY; ages 31-49, N=23); Middle-Older (MO; ages 50-66, N=21); Older Adult (OA; ages $67-85, N=19)$.

interaction term between condition, choice point, and age group was also significant $\left(F_{(17,453)}=2.11, P<0.01, \eta^{2}=0.05\right)$. Accuracy for NOL and OL mazes by choice point and age group are plotted in Figure 4B,C, respectively. This three-way interaction is critical, as it suggests that the effect of age group on accuracy depends on disambiguation demands (e.g., context-dependent choice points 2 and 3 for OL mazes vs. context-independent CP1 for both conditions and all choice points in NOL mazes; Fig. 4B,C; Supplemental Information, Table S3). Tukey post-hoc testing confirmed that at each choice point, there was no significant difference in accuracy between the three youngest groups in either the NOL and OL condition (all corrected Ps $>0.05$;). In contrast, the OA group performed significantly worse at $\mathrm{CP} 1$ and $\mathrm{CP} 2\left(\mathrm{CP} 1_{\mathrm{OL}}\right.$ mean $0.53 \pm$ $0.22 ; \mathrm{CP} 2_{\mathrm{OL} \text { mean }} 0.36 \pm 0.08 ; \mathrm{CP} 1_{\mathrm{NOL} \text { mean }} 0.50 \pm 0.14 ; \mathrm{CP} 2_{\mathrm{NOL}}$ mean $0.50 \pm 0.16)$ than both the $\mathrm{YA}\left(\mathrm{CP} 1_{\mathrm{OL}}\right.$ mean $0.80 \pm 0.14$; $\mathrm{CP} 2_{\mathrm{OL}}$ mean $0.67 \pm 0.09 ; \mathrm{CP} 1_{\mathrm{NOL}}$ mean $0.75 \pm 0.17 ; \mathrm{CP} 2_{\mathrm{NOL}}$ mean $0.79 \pm 0.17 ; P<0.05$ for all comparisons with $\mathrm{OA}$ ) and MY groups $\left(\mathrm{CP} 1_{\mathrm{OL}}\right.$ mean $0.76 \pm 0.20 ; \mathrm{CP} 2_{\mathrm{OL}}$ mean $0.61 \pm 0.21 ; \mathrm{CP} 1_{\mathrm{NOL}}$ mean $0.72 \pm 0.20 ; \mathrm{CP} 2_{\mathrm{NOL}}$ mean $0.72 \pm 0.23 ; P<0.05$ for all comparisons with $\mathrm{OA})$. For choice point 3 , the $\mathrm{OA}\left(\mathrm{CP} 3_{\mathrm{OL}}\right.$ mean $0.46 \pm 0.09$; $\mathrm{CP} 3_{\mathrm{NOL}}$ mean $\left.0.54 \pm 0.16\right)$ performed worse than the $\mathrm{YA}\left(\mathrm{CP} 3_{\mathrm{OL}}\right.$ mean $\left.0.64 \pm 0.20 ; \mathrm{CP} 3_{\mathrm{NOL} \text { mean }} 0.84 \pm 0.13\right), \mathrm{MY}\left(\mathrm{CP} 3_{\mathrm{OL} \text { mean }} 0.63 \pm\right.$ $\left.0.21 ; \mathrm{CP} 3_{\mathrm{NOL} \text { mean }} 0.76 \pm 0.24\right)$, and $\mathrm{MO}\left(\mathrm{CP} 3_{\mathrm{OL} \text { mean }} 0.56 \pm 0.17\right.$; $\mathrm{CP} 3_{\mathrm{NOL}}$ mean $0.70 \pm 0.21$ ), but this comparison did not reach significance after applying multiple comparison correction. For both conditions, the MO group performed worse than the YA and MY groups, but statistically did not differ from the other age groups at any choice point after applying multiple comparison correction $\left(\mathrm{CP} 1_{\mathrm{OL} \text { mean }} 0.67 \pm 0.19 ; \mathrm{CP} 2_{\mathrm{OL} \text { mean }} 0.48 \pm 0.15 ; \mathrm{CP} 1_{\mathrm{NOL} \text { mean }} 0.59\right.$ $\pm 0.24 ; \mathrm{CP} 2$ NOL mean $0.65 \pm 0.23 ; P>0.05$ for all comparisons to other age groups). Additionally, within each age group, noncritical choice points, that is, locations at which participants did not require disambiguation (NOL CP1, CP2, CP3, and OL CP 1), did not significantly differ from one another (all $P \mathrm{~s}<0.05$ ).

\section{Interactions between age and CRF on spatial disambiguation}

Next, we set out to elucidate how age and CRF may interactively predict performance on the spatial disambiguation task. These analyses were limited to participants who successfully completed the CRF assessment and RDT $(\mathrm{N}=62)$. Using multiple regression we entered age as a continuous variable, $\mathrm{CRF}$ as a continuous variable, condition (OL, NOL) and choice point (CP1, CP2, CP3) as main effects. In addition, we included an interaction term for age by CRF to determine how these two continuous variables may interactively predict accuracy, as well as an interaction term for condition by choice point, given the previous analyses indicating this as a significant interaction in predicting accuracy. As in the previous analyses, we found main effects of condition $\left(F_{(1,361)}=\right.$ $\left.20.41, P<0.001, \eta^{2}=0.036\right)$, choice point $\left(F_{(2,361)}=5.65, P<0.01\right.$, $\left.\eta^{2}=0.02\right)$ and age $\left(F_{(1,361)}=106.59, P<0.001, \eta^{2}=0.19\right)$, as well as a significant interaction between condition and choice point $\left(F_{(2,361)}=13.33, P<0.001, \eta^{2}=0.05\right)$ in this subsample. Critically, as predicted, we found a significant interaction between age and $\operatorname{CRF}\left(F_{(1,361)}=6.87, P<0.01, \eta^{2}=0.012\right)$, such that CRF attenuated age-related reductions on task accuracy as illustrated by flatter regression lines for groups with higher CRF (Fig. 5A).

Last, we sought to determine the effects of condition, choice point, age, and CRF on response time for the RDT, as previous studies have demonstrated a link between higher CRF and faster response times for cognitive tasks (Kramer et al., 1999). Initial response time analyses included all subjects $(\mathrm{N}=80)$ to characterize the main and interactive effects of condition, choice point and age on response time data. We entered response time data into a linear mixed model, specifying main effects of condition (OL, NOL), choice point (CP1, CP2, CP3), and age, as well as included all twoway interaction terms as fixed-effects, sex and education as covariates and subject as a random effect. Age significantly predicted response time $\left(F_{(1,76)}=57.72, P<0.001\right.$, Supplemental Information, Table S3), such that older adults had higher response times (i.e., slower responses) than younger adults. A main effect of condition demonstrated that participants responded significantly slower for OL than NOL mazes $\left(F_{(1,392)}=4.1, P<0.05\right)$. There was also a main effect of choice point $\left(F_{(2,390)}=18.21, P<0.001\right)$, with response times at choice point 1 being significantly longer than at choice points 2 or $3\left(\mathrm{CP} 1_{\text {mean }} \mathrm{RT}=1321.47 \pm 515.7 \mathrm{msec}\right.$; $\mathrm{CP} 2_{\text {mean }} \mathrm{RT}=838.42 \pm 411.3 \mathrm{msec} ; \mathrm{CP} 3_{\text {mean }} \mathrm{RT}=690.05 \pm 301.3$ msec). The interaction term between age and choice point was also significant $\left(F_{(2,76)}=57.72, P<0.001\right)$. No other main or interactive effects reached significance (all $P$ s $>0.5$ ). Next, we entered data from the participants that completed the CRF test into a multiple linear regression model to test the contribution of CRF to response time $(\mathrm{N}=62)$. Again, we included condition, choice point, age, and CRF as main effects. We specified interaction effects of condition by choice point as well as age by CRF. Results of this analysis demonstrated main effects of choice point $\left(F_{(2,361)}=\right.$ 104.9, $\left.P<0.001, \eta^{2}=0.29\right)$ and age $\left(F_{(1,361)}=143.5, P<0.001\right.$, $\left.\eta^{2}=0.20\right)$. Similar to the analysis on accuracy, we again found a 


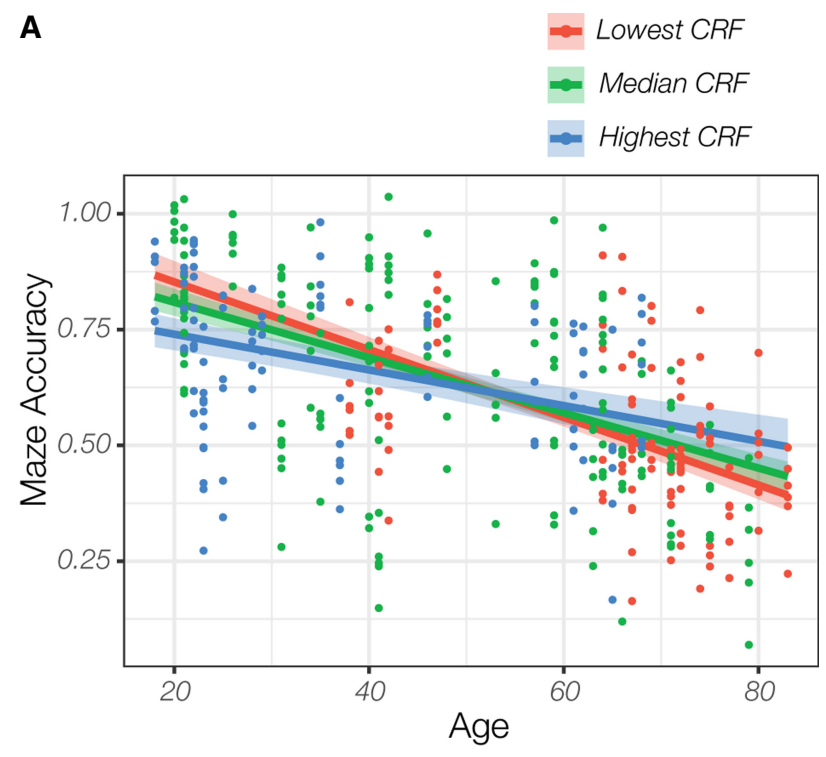

B

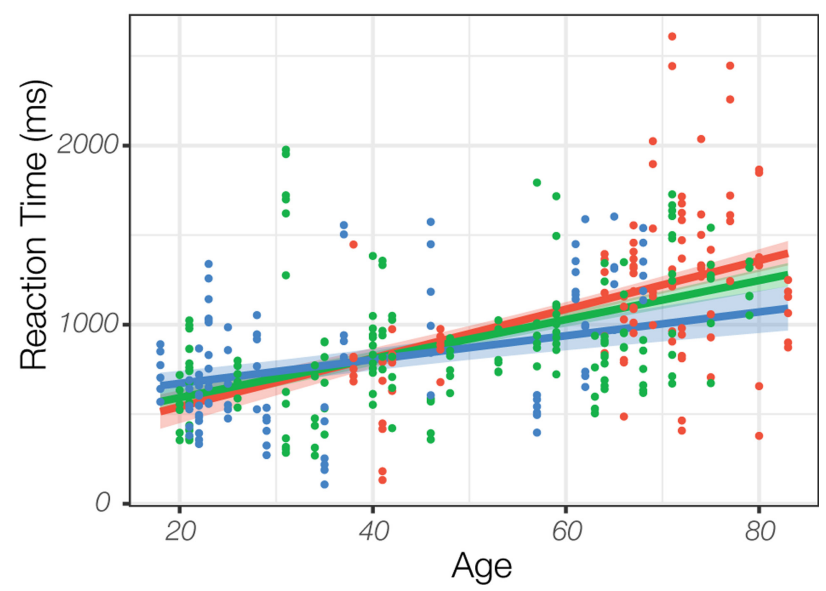

Figure 5. Age and CRF interactively predict performance on the RDT. (A) Higher CRF (represented by top 10\% CRF in blue) attenuates age-related impairment on accuracy, whereas lower CRF (represented by bottom 10\% CRF in red; green represents median CRF) is associated with pronounced age-related reduced task accuracy. (B) Similarly, higher CRF lessens age-related slowing in reaction time. Interactions are visualized in $\mathrm{R}$ using the visreg package (Breheny and Burchett 2016).

significant interaction between age by $\operatorname{CRF}\left(F_{(1,361)}=6.62, P<0.01\right.$, $\eta^{2}=0.01$ ) with response time data (Fig. 5B). Response time results are complementary to accuracy results, demonstrating a robust interaction between age and CRF on performance for the spatial maze task such that higher CRF attenuates age-related slowing on response time. Taken together, these results suggest that greater CRF can attenuate age-related reductions in task performance on the RDT, both in terms of accuracy and response time.

\section{Correlations between MST and RDT performance and neuropsychological tests}

Previous work has shown that the RAVLT scores correlate with LDI (Stark et al. 2013; Reagh and Yassa 2014; Stark and Stark 2017) suggesting RAVLT may also serve as a putative measure for mnemonic discrimination. We performed Spearman's correlations between performance on neuropsychological tests and performance on aspects of the MST (Lure Discrimination Index: LDI and
Recognition Memory: REC) and the RDT (OL maze accuracy, NOL maze accuracy), correcting for multiple comparisons using the Bonferroni method. These results are displayed in Table 2. LDI positively correlated with performance on all aspects of the RAVLT task $(P \mathrm{~s}<0.001)$. In contrast, REC did not correlate with any neuropsychological measures. In addition, accuracy for both OL and NOL mazes significantly positively correlated with all aspects of the RAVLT task. It is also of note that performance on the RDT also correlated with performance on the Victoria Stroop Test (VST). The VST is known to relate to response inhibition, which decreases with age (Strauss et al. 2006). It is possible that performance on the RDT requires response inhibition at critical choice points to allow flexible navigation. Performance on the Trail Making Test (TMT) and scores from the Santa Barbara Sense of Direction Scale (SBSOD) scale did not correlate with performance on either of the mnemonic discrimination tasks.

\section{Discussion}

In the current study, we sought to examine the interactive effects of age and CRF on mnemonic discrimination across the human adult lifespan. We tested the hypothesis that higher CRF attenuates age-related deficits in mnemonic discrimination both in the context of an object based mnemonic discrimination task and a more complex virtual navigation task requiring contextual disambiguation which likely relies on spatial pattern separation. We present robust negative age-related effects on mnemonic discrimination performance across both the nonspatial and spatial domains. Critically, we include individuals across the adult lifespan for a comprehensive examination of these age-effects on spatial and nonspatial mnemonic discrimination. Higher CRF did not seem to affect or attenuate age-related performance in the MST. Comparatively, results from the RDT confirm our hypothesis that higher CRF attenuates age-related performance decrements on spatial mnemonic discrimination. These data suggest that the route disambiguation task could serve as a highly sensitive metric for spatial pattern separation.

\section{Age negatively impacts behavioral performance on object and spatial mnemonic discrimination}

In Experiment 1, we presented an independent replication of age-related impairment of object-based nonspatial mnemonic discrimination using the MST (Holden et al. 2013; Stark et al. 2013, 2015). Our results mirror previous work showing that performance for mnemonic discrimination linearly decreases with age, whereas traditional recognition remains largely unaffected by age (Stark

Table 2. Correlations between neuropsychological tests and mnemonic discrimination performance

\begin{tabular}{lcrcc}
\hline & LDI & REC & OL & NOL \\
\hline Neuropsych & & & & \\
$\quad$ SBSOD & -0.147 & -0.075 & -0.126 & -0.010 \\
RAVLT Total & $0.456^{\star * *}$ & 0.134 & $0.481^{\star *}$ & $0.451^{\star *}$ \\
RAVLT Immediate & $0.452^{\star * *}$ & 0.158 & $0.416^{\star *}$ & $0.413^{* *}$ \\
RAVLT Delay & $0.482^{* * *}$ & 0.236 & $0.398^{*}$ & $0.408^{*}$ \\
TMT B/A Ratio & -0.001 & 0.062 & -0.278 & 0.007 \\
VST Ratio & -0.146 & 0.081 & $-0.358^{*}$ & $-0.361^{*}$ \\
\hline
\end{tabular}

Spearman's correlations between performance on neuropsychological tests and performance on aspects of the MST (Lure Discrimination, LDI; Recognition memory, REC) and the Route Disambiguation Task (OL maze accuracy, NOL maze accuracy). Values indicate Spearman's rho, and significance values were corrected for multiple comparisons with the Bonferroni method. $\left(^{*}\right) P<0.05$, $\left({ }^{* *}\right) P<0.01,\left({ }^{* *}\right) P<0.001$. 
et al. 2013). Our subsequent analyses focusing on the lure trials indicate a clear age-related shift in the response profile from correct identification of lure items as "similar" in the youngest adults to an incorrect identification of lure items as "old" in the older adult cohorts. The youngest cohort significantly outperformed all other groups in this respect. This strong preference for identifying lures as "similar" in the YA group was not apparent in the younger middle-aged group (MY). The MY participants were equally as likely to identify a lure as "similar" or commit a false positive error by identifying a lure as "old." Individuals in the two oldest groups committed this type of false positive significantly more often than the YA and MY groups. This indicates that age-related effects on mnemonic discrimination may take effect as early as younger middle-age, which in our study was defined as between 31-49 yr. Critically, all age groups reliably categorized new stimuli as "new" and repeated stimuli as "old," indicating a preferential effect of age on lure trials that cannot be explained more generally by age-related changes in recognition memory.

In Experiment 2, we used a task developed in our laboratory in which participants learned and subsequently retrieved OL and NOL routes through virtual mazes. Results demonstrated a robust deleterious linear age-dependent effect on task performance, similar to the age-effect found in the MST. The YA group performed best in both the OL and NOL conditions. Although the MY group did not statistically differ from the YA group, the MO group performed worse than the two younger groups, and the OA group showed robust negative age-related effects in both conditions. Importantly, our results suggest subtle age-related changes in spatial contextual disambiguation may occur as early as middle age. Across all age groups, performance was worse for choice points that required route disambiguation (i.e., OL critical choice points) than for those that did not, suggesting a deficit in spatial contextual discrimination. We also demonstrate a reduced age-effect in the spatial task for NOL mazes and, as stated above, the absence of an age-related effect in traditional recognition in the MST.

The results are in line with literature on deleterious age effects on episodic memory both in the spatial (Moffat and Resnick 2002; Erickson et al. 2009; Holden et al. 2012; Rodgers et al. 2012; Reagh et al. 2014; DeFord et al. 2020) and nonspatial domains (Yassa et al. 2011; Holden et al. 2013; Stark et al. 2013; Bernstein and McNally 2019). Importantly, within the same participants, we find comparable age-related effects on memory disambiguation performance in both spatial and nonspatial tasks. Although age effects on spatial pattern separation have not been as thoroughly studied across the adult lifespan, our results from Experiment 2 are consistent with previous studies comparing spatial pattern separation in younger and older adults (Stark et al. 2010; Holden and Gilbert 2012; Reagh et al. 2014). One recent study investigated mnemonic discrimination performance using two versions of the MST to compare between object and scene processing (Stark and Stark 2017). Consistent with our results across Experiments 1 and 2, they found that the older adults performed significantly poorer on mnemonic discrimination in both conditions. Our results are also in accordance with a recent study by Williams and colleagues that demonstrated that middle-aged adults performed more poorly relative to young adults on a delayed nonmatch to sample spatial pattern separation task with varying levels of spatial distance (Williams et al. 2019). In that study, young adults significantly outperformed middle-aged adults, who, in turn, outperformed older adults on low spatial similarity (i.e., requiring less pattern separation). Interestingly, middle-aged and older adults performed equally on trials with high spatial similarity (i.e., requiring more pattern separation), whereas younger adults performed significantly better on the same trials. Here, we demonstrate robust age effects in both tasks, suggesting the impact of age on mnemonic discrimination is domain-independent.
Our findings are consistent with computational and rodent models of pattern separation. Neurocomputational modeling suggests pattern separation may depend on the newborn neurons in the dentate gyrus of the hippocampus promoting disambiguation of highly similar information into individually stored representations (Marr 1971; O'Reilly and McClelland 1994; Aimone et al. $2006,2009)$. In aging rodents, there is a drastic decrease in the amount of AHN within the dentate gyrus (Kuhn et al. 1996), and this reduction is associated with impaired behavioral pattern separation performance (Creer et al. 2010; Sahay et al. 2011). Although AHN cannot be studies in living humans, our results from both Experiment 1 and Experiment 2 are predicted by these models.

\section{Higher CRF significantly attenuates age effects in spatial mnemonic discrimination, but fails to affect object mnemonic discrimination performance}

A primary goal of the current study was to investigate the interactive effects of age and CRF on mnemonic discrimination. In the MST, CRF level failed to affect behavioral performance across any age group measured both by LDI and the response profile. Higher CRF did not seem to affect classification accuracy for lures overall, and did not rescue or affect the false positive error rate on this task for aged individuals. This suggests that CRF itself may not influence performance on this task. The lack of effect of CRF level on behavioral performance is in contrast with a recent report that higher-fit young adults significantly outperformed lower-fit young adults on the MST (Suwabe et al. 2017a). In that experiment, the results from an investigation of 75 young adult participants demonstrated that higher-fit young adults correctly classified lure trials as "similar" significantly more than lower-fit young adults, but this result was limited only to trials where the objects were considered moderately similar. Suwabe and colleagues did not find an effect fitness level on lure categorization in low or high similarity trials (2017). One possibility for this discrepancy is that our study was designed to examine the interactive effects between CRF and aging, did not focus solely on young adults and had a substantially smaller number of young adult participants. Our smaller sample size could be contributing to the lack of CRF effects on behavior for this age group. Subtle enhancement of MST performance has also been demonstrated in young adults following acute bouts of aerobic exercise (Suwabe et al. 2017b). Results from these previous studies were limited to younger adult populations given older adults were not included. Although these previous reports have demonstrated improvements in this task following acute and longterm exercise in young adults, our results suggest that CRF level alone does not significantly predict performance in a large sample across the lifespan. The current study extended these previous reports by examining associations between CRF and behavioral pattern separation across the adult lifespan.

In Experiment 2, we used the RDT in which participants learned and subsequently retrieved OL and NOL virtual mazes. Critically, higher CRF appeared to partially attenuate the agerelated effects both for task accuracy and response time, adding evidence to a growing body of literature that greater fitness is neuroprotective against age-associated spatial memory deficits. Importantly, the RDT relies on many of the same brain regions as object-based mnemonic discrimination tasks. The MST has primarily been investigated using fMRI focusing on the differential contributions of the hippocampal subfields to mnemonic discrimination, most notably the DG/CA3 (Bakker et al. 2008). Previous fMRI experiments from our laboratory using the RDT established that OL mazes evoked stronger activation of the hippocampus and parahippocampal cortex bilaterally than NOL mazes during the cue period of the maze prior to the start of navigation (Brown et al. 2010). Navigating through the OL hallway increased 
activation in the right hippocampus, parahippocampal cortex, and the orbitofrontal cortex, comprising a navigational circuitry that support spatial contextual disambiguation. A follow-up study using the RDT with high-resolution fMRI focused on the medial temporal lobes and confirmed greater activation specifically in the DG/ CA3 and CA1 subregions in OL mazes compared to NOL mazes (Brown et al. 2014). In addition, activation in the entorhinal cortex and CA1 during learning of new OL mazes was predictive of subsequent navigation performance. Importantly CA1 has robust and direct connections to the medial and orbitofrontal cortices which, in turn, connect to medial entorhinal cortex (van Strien et al. 2009). This highlights the importance of this circuitry in supporting contextual spatial disambiguation. Future studies should examine the interaction between CRF and age on the fMRI signal in the circuitry underlying contextual spatial disambiguation.

Here, we sought to elucidate potential domain-dependent relationships between CRF and pattern separation in humans across the adult lifespan. We hypothesized that greater CRF would enhance mnemonic discrimination in both domains. Additionally, we predicted CRF would modulate the relationship between age and task performance. Although results from Experiment 2 confirm our predictions, results from Experiment 1 did not show the hypothesized age-related attenuation effect for higher CRF in the MST. One possibility for this difference is that there are multiple mechanisms by which CRF is impacting the brain and affecting cognition. One of the foremost theories on the mechanism by which CRF and exercise strengthens mnemonic discrimination is by altering hippocampal circuitry and enhancing pattern separation. In animal models, aerobic exercise reliably increases AHN in rodents (van Praag et al. 1999, 2005), attenuates age-related decline of neurogenesis (van Praag et al. 2005), in turn promoting pattern separation performance on spatial tasks in rodents (Creer et al. 2010). Studies in rodents linking AHN, aging, and pattern separation are very difficult to perform without a spatial component. In humans, greater CRF and improving CRF with exercise have been linked to both higher bilateral hippocampal gray matter volume (Erickson et al. 2009, 2011; Herting and Nagel 2012; Varma et al. 2015; Rosano et al. 2017) as well as better performance on spatial working memory tasks in older adults (Erickson et al. 2009, 2011). Our results suggest that CRF attenuates age-effects in spatial mnemonic discrimination and are in line with previous studies in both rodents and humans.

However, the existence of AHN in the human brain is heavily debated (Eriksson et al. 1998; Boldrini et al. 2018; Sorrells et al. 2018), and it is possible that basal levels of newborn neurons thought to perform pattern separation does not correlate with CRF level in humans. Object-based mnemonic discrimination in young adults has been shown to be enhanced following a very short bout of light intensity aerobic activity, possibly via facilitation of rapid increased functional connectivity between the DG/ CA3 and neocortex (Suwabe et al. 2018) in healthy young adults. Beyond the hippocampus, there are functional dissociations between regions within the entorhinal cortex relating to object or spatial mnemonic discrimination (Reagh and Yassa 2014), as well as known age-related differences engaging these pathways (Reagh et al. 2018). The entorhinal cortex is a crucial structure supporting spatial memory and navigation (Hafting et al. 2005) and together with the hippocampus supports disambiguation of OL stimuli during learning (Newmark et al. 2013). Both the volume (Raz et al. 2004) and connectivity (Yassa et al. 2010a) between these two structures are negatively affected by aging. In contrast, work by our group has previously demonstrated a positive relationship between higher CRF and volume in the entorhinal cortex in young adults (Whiteman et al. 2016). Therefore, it is likely that CRF may counteract deleterious aging effects in the entorhinal-hippocampal-neocortical circuitry and facilitate spatial memory and navigation. This interpretation is consistent with our results, which suggest that higher CRF may attenuate the deleterious effects of aging on spatial mnemonic discrimination. Given the multidimensional effects of exercise on the brain, we suggest future studies focus on elucidating whether CRF and change in CRF following exercise training modulates connectivity between the entorhinal-hippocampal circuitry as well as the hippocampus and the neocortex, and how this modulation may change across the adult lifespan.

Previous studies have also demonstrated that RAVLT Delay scores correlate with LDI (Stark et al. 2013; Reagh and Yassa 2014; Stark and Stark 2017) and DG/CA3 volume (Stark and Stark 2017), suggesting RAVLT is dependent on the hippocampus and may also serve as a putative measure for mnemonic discrimination. Here, we replicate the findings that LDI correlated with performance on all aspects of the RAVLT task. Furthermore, we demonstrated that RAVLT performance also significantly correlated with accuracy on the RDT, adding further evidence that performance on the RDT and MST may be similarly supported. In contrast to the LDI and RDT, REC did not correlate with any neuropsychological measures.

We acknowledge several limitations to our study. First, we acknowledge a potential selection bias toward higher fit older adult participants. Due to the medical and safety considerations associated with performing a fitness test, particularly in older adults, inclusion and exclusion criteria strictly narrowed the potential participants that could be included in this study. Although the CRF levels for our younger adults (YA and MY) distribute closely around the age and sex-corrected mean (Supplemental Information, Fig. S1), there is a selection bias with older adults. In comparison to age and sex stratified normative data from the American College of Sports Medicine, our older adults have a higher than average fitness for their age group. This is because lower-fit older adults we screened and precluded for participation in the current study were often prescribed certain medications that alter heart rate (e.g., cardioactive medication) and had several comorbid disorders (e.g., heart, lung, or musculoskeletal conditions) that would make participation in an aerobic fitness test difficult and would have placed these individuals at a higher risk for an adverse event. Future studies should aim to include lower-fit elderly individuals under the supervision of a trained medical professional to ensure participant safety. This selection bias for higher-fit older adults could have potentially affected the significance of the results regarding CRF attenuation of age-related performance reductions on these tasks, particularly for the MST where we did not observe CRF effects. Despite this, we still observed robust age-related effects in both Experiment 1 and 2, as well as a robust interaction between CRF and age on performance in Experiment 2. It is also important to recognize the relatively small sample size per age group when comparing higher and lower-fit individuals. It is possible this may have contributed to the lack of effect of higher CRF on mnemonic discrimination across the age groups in Experiment 1 . An additional limitation is the cross-sectional nature of the current study, making it difficult to separate cohort effects from true age effects. We cannot address inter-individual variability that may have been introduced by selection bias and our CRF estimation. Future fitness intervention studies should aim to elucidate how improving CRF in initially low-fit adults could differentially affect performance on each mnemonic discrimination task and should include functional and structural neuroimaging measures as outcomes.

Here, we offer a unique contribution to the literature by including participants across the adult lifespan and investigating how age and CRF interact to impact both spatial and nonspatial mnemonic discrimination task performance. The majority of previous literature on the effects of CRF on cognition compare younger and older adults, largely excluding the middle-aged population. 
Critically, we include individuals across the adult lifespan, which may be crucial as previous work has suggested that middle-age may be the start of subtle cognitive and structural change predictive of future neurodegenerative changes (Clark et al. 2016; Melah et al. 2016) and may be the ideal time to implement neuroprotective interventions such as aerobic exercise (Tolppanen et al. 2014). We conclude that higher CRF mitigates age-related performance reductions in a virtual spatial contextual discrimination task but not nonspatial mnemonic discrimination in our lifespan sample. This suggests that higher CRF may lead to cognitive reserve across the adult lifespan, particularly for spatial disambiguation of similar contexts. Performance on spatial mnemonic discrimination tasks may be a useful tool to track vulnerability in older individuals sensitive to cognitive decline. Future work should investigate the potential for aerobic exercise as a therapeutic strategy for promoting mnemonic flexibility across domains. Mnemonic discrimination tasks, such as the MST and RDT utilized in this study, may serve as sensitive metrics in these future experiments.

\section{Materials and Methods}

\section{Participants}

Participants were recruited from the Boston community via flyers, online advertisement, and advertisements in a local paper. Interested individuals were prescreened for the following conditions: history of neurological or psychiatric conditions; learning disability; heart, lung, or musculoskeletal conditions or disorders; thyroid disease; diagnosis of kidney failure; diabetes mellitus; electrolyte disorder; high cholesterol; eating disorder or obesity; pregnancy; cancer; current use of any cardioactive or psychoactive medication, or recreational smoking. For inclusion in the study, individuals needed to be between the ages of $18-85$ yr old. Following the phone prescreening, 102 participants came to the laboratory for an initial study visit for additional screening. Participants were excluded if they met the following three criteria for obesity: BMI greater than or equal to 35 for both men and women; waist circumference of greater than $88 \mathrm{~cm}$ for men and $102 \mathrm{~cm}$ for women; a waist-to-hip ratio greater than or equal to 0.95 for men between the ages of 18-54 yr, 1.03 for men over $54 \mathrm{yr}, 0.86$ for women between the ages 18-54 yr, 0.90 for women older than $54 \mathrm{yr}$. Individuals older than $55 \mathrm{yr}$ also were administered the Dementia Rating Scale (DRS-2) and were excluded if they received an eight or below for the age and education corrected scaled score. Fifteen subjects were excluded following the screening visit, seven withdrew voluntarily or were lost to contact. A total of eighty participants completed the second visit. Data from two participants on the Mnemonic Similarity Task (MST) were not included in analyses due to computer malfunction, leaving a final data set from 78 participants. All eighty participants are included in analyses for the Route Disambiguation Task (RDT). Sixty-two participants successfully completed the aerobic fitness assessment, and their data is included in all analyses elucidating CRF as a predictor of behavior. In an effort to elucidate if there is a specific effect of age, or an interactive effect between age and CRF on task performance, we chose to bin our data into four age groups with the goal of relatively equal statistical power per age group. Details of participant characteristics are presented in Table 1. All participants were fluent English speakers, all had normal or corrected to normal vision, and all gave signed, informed consent before participating in the experiments. All protocols were approved by the Boston University Charles River Campus Institutional Review Board.

\section{Study procedure}

For each participant, the experiment consisted of two study visits: (1) informed consent and screening, (2) cognitive testing on both the spatial and nonspatial tasks followed by a submaximal treadmill test to measure CRF. Both visits were conducted within 3 wk for each individual subject. See Figure 1A for study procedure and experimental timeline.
Subsequent to signed, informed consent at the start of the first study visit, participants were formally screened for the above prescreening criteria, and anthropometric measurements were taken. Screening ensured participants were able to safely perform the CRF test. Resting heart rate was taken using a chest strap (Polar H7) wirelessly connected to a HR monitor watch (Polar m400). Blood pressure was measured manually with an inflatable sphygmomanometer. Height and weight were measured to calculate body mass index (BMI); waist and hip circumference measurements were also taken. During the initial visit, participants also completed a neuropsychological assessment, including the following: Rey Auditory Verbal Learning Test (RAVLT); Santa Barbara Sense of Direction Scale (SBSOD); Trail Making Test A/B (TMT); Victoria Stroop Test (VST). Behavioral performance data from the neuropsychological tests can be found in Tables 1,2 and in Supplemental Information.

The second study visit lasted $\sim 2.5 \mathrm{~h}$, during which participants completed both the cognitive tasks and underwent a CRF test. Visit 2 began with instructions and training for the RDT. Once participants reached criterion on all mazes (see Experiment 2: Route Disambiguation Task for details), the incidental encoding and retrieval phases (see below) of the MST were administered in serial fashion. Following the retrieval phase of the MST, participants completed eight runs of the RDT. After the cognitive testing protocol was completed, participants were fitted for heart rate monitors and began the CRF test.

\section{Experiment 1: Mnemonic Similarity Task (MST)}

The MST has been established as a reliable way to assess pattern separation behavior in humans (Stark et al. 2013). Task administration occurs over two phases; incidental encoding and retrieval. During the encoding phase, the participants were shown 128 pictures of everyday objects on a computer screen and were asked to make an indoor/outdoor judgment for each photo using keys on a computer. The images were displayed for $2 \mathrm{sec}$ with a $0.5 \mathrm{sec}$ ITI as done in previous work (Stark et al., 2013). Immediately following the encoding phase, participants were asked to perform a surprise memory test. They were shown video instructions to explain the task in which they were told that for each item they will see, they are to identify the item as "Old," "Similar," or "New" via button press (192 items total). "Old" designations are for items that were identical to an image shown during the indoor/outdoor task (64 total repeat items; Target). "New" designations are for items not previously seen (64 Foils). "Similar" designations are to be given to items that were similar to those shown during the indoor/outdoor task, but not identical (64 Lures).

\section{Experiment 2: Route Disambiguation Task (RDT)}

This task was adapted from previous studies (Brown et al., 2010, 2012, 2014). Participants learned to navigate through eight virtual mazes from a ground-level first-person perspective using a desktop computer. Each maze consisted of three hallways and three intersections. Each intersection was marked with unique objects to distinguish it from other intersections. The eight mazes were evenly divided into two conditions, including an OL and NOL condition (see Fig. 1C). The four mazes in the OL condition consisted of two pairs of mazes that began and ended in distinct locations but converged in the middle to share a middle hallway with its respective paired maze. The NOL condition consisted of four distinct mazes that did not have OL elements or locations. Therefore, the intersections in the NOL mazes required the same navigational choices for successful navigation in each trial. For the OL mazes, the starting intersection also consistently was associated with the same navigational choice in each trial. In contrast, the second and third intersections in the OL mazes were shared across the OL maze pairs. Therefore, the correct response at these critical choice points were dependent on which location the maze was entered from (e.g., the axes or the rubber ducky in Fig. 1C), and is thought to rely, at least in part, on spatial pattern separation. At every intersection the participant pressed designated keys on the number pad of 
the keyboard to turn "left, continue straight, or go right," selecting the subsequent hallway in the sequence of spatial locations within the maze. Participants were not able to navigate in a reverse direction. Once a choice was indicated, subjects were propelled forward at a fixed speed to the next intersection.

Participants underwent maze training to become familiar with the environments. Prior to learning the mazes, participants were given explicit instructions and guided through two OL demonstration mazes, allowing them to become familiar with navigating in the environment and to ensure they understood the mechanics of the task. The demonstration mazes were distinct from the mazes used in the actual task. During task training, participants navigated through each of the eight mazes in a serial fashion, and had to successfully navigate each maze to $100 \%$ accuracy three consecutive times in order to proceed to the next maze. The order of the maze training was randomized across participants.

Participants learned to navigate the virtual mazes by receiving feedback for their navigational choices. When a participant made a wrong turn, the computer provided feedback by identifying the correct response for that intersection, and passively moved the participant in the correct direction. When the participant chose correctly, he or she proceeded in the correct direction without additional feedback. If the participant failed to make a response in the allotted time $(2 \mathrm{sec})$, the computer indicated that no response was made and identified the correct response and passively moved the participant in the correct direction. Any intersection where the participant failed to make a response was treated as an incorrect response. Lastly, participants were given one practice run similar to those given in the testing session. During the practice run, all eight mazes were presented in a random order within the practice run. Feedback was provided during training as well as during practice and test runs. Following a 20-min delay after completing the practice run, during which participants participated in the MST, participants performed eight test runs of the RDT. Each run contained all eight mazes presented in a random order (64 trials total). Accuracy and response time were recorded using E-prime 2.0. Run order was counterbalanced across participants.

\section{Neuropsychological assessment}

All participants were administered the Rey Auditory Verbal Learning Test (RAVLT) to assess memory, Victoria Stroop Test (VST; Strauss et al. 2006) to assess response inhibition, and Trail Making Test A and B (TMT; Tombaugh 2004; Strauss et al. 2006) to assess processing speed and executive functioning, respectively. Participants completed the Santa Barbara Sense of Direction Scale (SBSOD; Hegarty et al. 2002) to provide a self-reported measure of environmental spatial ability. TMT ratio was calculated by time in seconds to complete TMT B divided by time in seconds to complete TMT A. VST ratio was calculated by time in seconds for the interference condition divided by time in seconds for the dots condition. Lower scores on both indicate higher executive switching and better response inhibition.

\section{Cardiorespiratory Fitness (CRF) test}

Participants were instructed to refrain from strenuous physical activity for $24 \mathrm{~h}$ prior to the visit and instructed to eliminate consumption of caffeine for $3 \mathrm{~h}$ prior to the CRF assessment. In order to avoid acute effects of exercise on cognition, participants performed the CRF assessment following cognitive testing. Immediately before CRF testing, participants were fitted with a chest strap to monitor heart rate (HR) (Polar, H7). The chest strap was wirelessly connected to a HR watch (Polar m400). Seated resting HR measurements as well as standing resting HR measurements were recorded (see Table 1). Resting blood pressure was measured manually with an inflatable sphygmomanometer immediately prior to and following the CRF assessment. Briefly, participants completed a submaximal-graded exercise test on a motor-driven treadmill (Precor TRM835) using a modified Balke protocol to assess CRF (Hagberg 1994; Cooper and Storer 2001; ACSM 2014) as described in further details in Supplemental Information. CRF was operationalized as estimated $\mathrm{VO}_{2 \mathrm{MAX}}$. Eighteen partic- ipants did not reach termination criteria (see Supplemental Information), leaving 62 participants with CRF data for inclusion in analyses.

\section{Statistical analyses}

All statistical tests were conducted using R (3.5.0). For models elucidating the effect of age on cognition, analyses included valid cognitive data from all participants ( $\mathrm{N}=78$ for MST, $\mathrm{N}=80$ for RDT). Analyses including CRF as a predictor of cognition included data from participants that successfully completed the CRF assessment ( $\mathrm{N}=60$ for MST; $\mathrm{N}=62$ for RDT). Our statistical analysis approach was motivated by a desire to replicate the age-effects on MST performance as described in Stark et al. (2013). Therefore, we included Pearson correlations, multiple regression, and ANOVAs by age group. For initial comparisons of age and CRF level on cognition, all variables were treated as continuous and simple correlations were performed using Pearson's $r$. Multiple linear regression and linear mixed-effects models were used for subsequent investigation and included sex and education as covariates. For determining age group differences, ANOVA and repeated measures (rm-ANOVA) tests were used in addition to Tukey HSD post-hoc testing for subsequent comparisons where appropriate.

\section{Competing interest statement}

The authors declare that they have no conflict of interest with respect to this research, authorship, and/or publication of this article.

\section{Acknowledgments}

Research reported in this publication was supported by the National Institute On Aging of the National Institutes of Health under Award Number F31AG055262 (R.K.N.) and R00AG036845 (K.S.), and a Clara Mayo Memorial Research Fellowship (R.K.N.) from the Department of Psychological and Brain Sciences, Boston University. The authors would like to thank Tajalane Firmin and Sheila Yee for their assistance with data collection.

Author contribution: R.K.N., K.S., and C.E.S designed research; R.K.N. performed research, R.K.N. analyzed data; R.K.N., K.S., and C.E.S. wrote the paper; K.S. and C.E.S. oversaw the project.

\section{References}

ACSM. 2014. Guidelines for exercise testing and prescription, 9th ed (ed. Pescatello LS, Arena R, Riebe D, Thompson P). Wolters Kluwer/Lippincott Williams \& Wilkins Health, Philadelphia.

Aimone JB, Wiles J, Gage FH. 2006. Potential role for adult neurogenesis in the encoding of time in new memories. Nat Neurosci 9: 723-727. doi:10 .1038/nn1707

Aimone JB, Wiles J, Gage FH. 2009. Computational influence of adult neurogenesis on memory encoding. Neuron 61: 187-202. doi:10.1016/j .neuron.2008.11.026

Bakker A, Kirwan CB, Miller M, Stark CEL. 2008. Pattern separation in the human hippocampal CA3 and dentate gyrus. Science 319: 1640-1642. doi:10.1126/science.1152882

Barnes DE, Yaffe K, Satariano WA, Tager IB. 2003. A longitudinal study of cardiorespiratory fitness and cognitive function in healthy older adults. $J$ Am Geriatr Soc 51: 459-465. doi:10.1046/j.1532-5415.2003.51153.x

Bernstein EE, McNally RJ. 2019. Examining the effects of exercise on pattern separation and the moderating effects of mood symptoms. Behav Ther 50: $582-593$. doi:10.1016/j.beth.2018.09.007

Boldrini M, Fulmore CA, Tartt AN, Simeon LR, Pavlova I, Poposka V, Rosoklija GB, Stankov A, Arango V, Dwork AJ, et al. 2018. Human hippocampal neurogenesis persists throughout aging. Cell Stem Cell 22: 589-599. doi:10.1016/j.stem.2018.03.015

Breheny P, Burchett W. 2016. Package 'visreg' : Visualization of Regression Models, 9(December), 14. Retrieved from http://myweb.uiowa.edu/ pbreheny/publications/visreg.pdf

Brown TI, Stern CE. 2014. Contributions of medial temporal lobe and striatal memory systems to learning and retrieving overlapping spatial memories. Cereb Cortex 24: 1906-1922. doi:10.1093/cercor/bht041

Brown TI, Ross RS, Keller JB, Hasselmo ME, Stern CE. 2010. Which way was I going? Contextual retrieval supports the disambiguation of well learned 
overlapping navigational routes. J Neurosci 30: 7414-7422. doi:10.1523/ JNEUROSCI.6021-09.2010

Brown TI, Ross RS, Tobyne SM, Stern CE. 2012. Cooperative interactions between hippocampal and striatal systems support flexible navigation. Neuroimage 60: 1316-1330. doi:10.1016/j.neuroimage.2012.01.046

Brown BM, Peiffer JJ, Martins RN. 2013. Multiple effects of physical activity on molecular and cognitive signs of brain aging: can exercise slow neurodegeneration and delay Alzheimer's disease? Mol Psychiatry 18: 864-874. doi:10.1038/mp.2012.162

Brown TI, Hasselmo ME, Stern CE. 2014. A high resolution study of hippocampal and medial temporal lobe correlates of spatial context and prospective overlapping route memory. Hippocampus 24: 819-839. doi:10.1002/hipo.22273

Burns JM, Cronk BB, Anderson HS, Donnelly JE, Thomas GP, Harsha A, Brooks WM, Swerdlow RH. 2008. Cardiorespiratory fitness and brain atrophy in early Alzheimer disease. Neurology 71: 210-216. doi:10.1212/ 01.wnl.0000317094.86209.cb

Clark LR, Racine AM, Koscik RL, Okonkwo OC, Engelman CD, Carlsson CM, Johnson SC. 2016. Beta-amyloid and cognitive decline in late middle age: findings from the Wisconsin Registry for Alzheimer's Prevention study. Alzheimer's \& Dement 12: 805-814. doi:10.1016/j.jalz.2015.12 .009

Cooper CB, Storer TW. 2001. Exercise testing and interpretation: a practical approach. Cambridge University Press, New York, NY.

Creer DJ, Romberg C, Saksida LM, van Praag H, Bussey TJ. 2010. Running enhances spatial pattern separation in mice. Proc Natl Acad Sci 107: 2367-2372. doi:10.1073/pnas.0911725107

DeFord NE, DeJesus SY, Holden HM, Graves LV, Lopez FV, Gilbert PE. 2020. Young and older adults may utilize different cognitive abilities when performing a spatial recognition memory test with varying levels of similarity. Int J Aging Hum Dev 90: 65-83. doi:10.1177/ 0091415019831443

Déry N, Pilgrim M, Gibala M, Gillen J, Wojtowicz JM, Macqueen G, Becker S. 2013. Adult hippocampal neurogenesis reduces memory interference in humans: opposing effects of aerobic exercise and depression. Front Neurosci 7: 66. doi:10.3389/fnins.2013.00066

Doeller CF, Barry C, Burgess N. 2010. Evidence for grid cells in a human memory network. Nature 463: 657-661. doi:10.1038/nature08704

Erickson KI, Prakash RS, Voss MW, Chaddock L, Hu L, Morris KS, White SM, Wójcicki TR, McAuley E, Kramer AF. 2009. Aerobic fitness is associated with hippocampal volume in elderly humans. Hippocampus 19: 10301039. doi:10.1002/hipo.20547

Erickson KI, Voss MW, Prakash RS, Basak C, Szabo A, Chaddock L, Kim JS, Heo S, Alves H, White SM, et al. 2011. Exercise training increases size of hippocampus and improves memory. Proc Natl Acad Sci 108: 30173022. doi:10.1073/pnas.1015950108

Eriksson PS, Perfilieva E, Björk-Eriksson T, Alborn AM, Nordborg C, Peterson DA, Gage FH. 1998. Neurogenesis in the adult human hippocampus. Nat Med 4: 1313-1317. doi:10.1038/3305

Geinisman Y, DeToledo-Morrell L, Morrell F, Persina IS, Rossi M. 1992. Age-related loss of axospinous synapses formed by two afferent systems in the rat dentate gyrus as revealed by the unbiased stereological dissector technique. Hippocampus 2: 437-444. doi:10.1002/hipo .450020411

Hafting T, Fyhn M, Molden S, Moser M-B, Moser EI. 2005. Microstructure of a spatial map in the entorhinal cortex. Nature 436: 801-806. doi:10 $.1038 /$ nature03721

Hagberg JM. 1994. Exercise assessment of arthritic and elderly individuals. Bailliere's Clin Rheumatol 8: 29-52. doi:10.1016/S0950-3579(05)80223-7

Hegarty M, Richardson AE, Montello DR, Lovelace K, Subbiah I. 2002. Development of a self-report measure of environmental spatial ability. Intelligence 30: 425-448. doi:10.1016/S0160-2896(02)00116-2

Herting MM, Nagel BJ. 2012. Aerobic fitness relates to learning on a virtual Morris Water Task and hippocampal volume in adolescents. Behav Brain Res 233: 517-525. doi:10.1016/j.bbr.2012.05.012

Holden HM, Gilbert PE. 2012. Less efficient pattern separation may contribute to age-related spatial memory deficits. Front Aging Neurosci 4: 9. doi:10.3389/fnagi.2012.00009

Holden HM, Hoebel C, Loftis K, Gilbert PE. 2012. Spatial pattern separation in cognitively normal young and older adults. Hippocampus 22: 18261832. doi: $10.1002 /$ hipo. 22017

Holden HM, Toner C, Pirogovsky E, Kirwan CB, Gilbert PE. 2013. Visual object pattern separation varies in older adults. Learn Mem 20: 358-362. doi:10.1101/lm.030171.112

Holzschneider K, Wolbers T, Röder B, Hötting K. 2012. Cardiovascular fitness modulates brain activation associated with spatial learning. Neuroimage 59: 3003-3014. doi:10.1016/j.neuroimage.2011.10.021

Honea RA, Thomas GP, Harsha A, Anderson HS, Donnelly JE, Brooks WM, Burns JM. 2009. Cardiorespiratory fitness and preserved medial temporal lobe volume in Alzheimer disease. Alzheimer Dis Assoc Disord 23: 188-197. doi:10.1097/WAD.0b013e31819cb8a2
Jacobs J, Weidemann CT, Miller JF, Solway A, Burke JF, Wei X-X, Suthana N, Sperling MR, Sharan AD, Fried I, et al. 2013. Direct recordings of grid-like neuronal activity in human spatial navigation. Nat Neurosci 16: 11881190. doi:10.1038/nn.3466

Killian NJ, Jutras MJ, Buffalo EA. 2012. A map of visual space in the primate entorhinal cortex. Nature 491: 761-764. doi:10.1038/nature11587

Kirwan CB, Jones CK, Miller MI, Stark CEL. 2007. High-resolution fMRI investigation of the medial temporal lobe. Hum Brain Mapp 28: 959-966. doi: $10.1002 / \mathrm{hbm} .20331$

Kirwan BC, Hartshorn A, Stark SM, Goodrich-Hunsaker NJ, Hopkins RO, Stark CEL. 2012. Pattern separation deficits following damage to the hippocampus. Neuropsychologia 50: 2408-2414. doi:10.1016/j .neuropsychologia.2012.06.011

Kramer AF, Hahn S, Cohen NJ, Banich MT, McAuley E, Harrison CR, Chason J, Vakil E, Bardell L, Boileau RA, et al. 1999. Ageing, fitness and neurocognitive function. Nature 400: $418-419$. doi:10.1038/22682

Kuhn HG, Dickinson-Anson H, Gage FH. 1996. Neurogenesis in the dentate gyrus of the adult rat: age-related decrease of neuronal progenitor proliferation. J Neurosci 16: 2027-2033. doi:10.1523/JNEUROSCI .16-06-02027.1996

Lautenschlager NT, Cox K, Cyarto EV. 2012. The influence of exercise on brain aging and dementia. Biochim Biophys Acta 1822: 474-481. doi:10 1016/j.bbadis.2011.07.010

Ly M, Murray E, Yassa MA. 2013. Perceptual versus conceptual interference and pattern separation of verbal stimuli in young and older adults. Hippocampus 23: 425-430. doi:10.1002/hipo.22110

Marr D. 1971. Simple memory: a theory for archicortex. Philos Trans $R$ Soc Lond B Biol Sci 262: 23-81. doi:10.1098/rstb.1971.0078

McNaughton BL, Battaglia FP, Jensen O, Moser EI, Moser M-B. 2006. Path integration and the neural basis of the "cognitive map". Nat Rev Neurosci 7: 663-678. doi:10.1038/nrn1932

Melah KE, Lu SY-F, Hoscheidt SM, Alexander AL, Adluru N, Destiche DJ, Carlsson CM, Zetterberg H, Blennow K, Okonkwo OC, et al. 2016 Cerebrospinal fluid markers of Alzheimer's disease pathology and microglial activation are associated with altered white matter microstructure in asymptomatic adults at risk for Alzheimer's disease. $J$ Alzheimer's Dis 50: $873-886$. doi:10.3233/JAD-150897

Moffat SD, Resnick SM. 2002. Effects of age on virtual environment place navigation and allocentric cognitive mapping. Behav Neurosci 116: 851859. doi:10.1037/0735-7044.116.5.851

Moffat SD, Elkins W, Resnick SM. 2006. Age differences in the neural systems supporting human allocentric spatial navigation. Neurobiol Aging 27: 965-972. doi:10.1016/j.neurobiolaging.2005.05.011

Newmark RE, Schon K, Ross RS, Stern CE. 2013. Contributions of the hippocampal subfields and entorhinal cortex to disambiguation during working memory. Hippocampus 23: 467-475. doi:10.1002/hipo.22106

Nokia MS, Lensu S, Ahtiainen JP, Johansson PP, Koch LG, Britton SL, Kainulainen H. 2016. Physical exercise increases adult hippocampal neurogenesis in male rats provided it is aerobic and sustained. J Physiol 594: $1855-1873$. doi: $10.1113 / J P 271552$

O'Reilly RC, McClelland JL. 1994. Hippocampal conjunctive encoding, storage, and recall: avoiding a trade-off. Hippocampus 4: 661-682. doi:10 $.1002 /$ hipo.450040605

Pereira AC, Huddleston DE, Brickman AM, Sosunov AA, Hen R, McKhann GM, Sloan R, Gage FH, Brown TR, Small SA. 2007. An in vivo correlate of exercise-induced neurogenesis in the adult dentate gyrus. Proc Natl Acad Sci 104: 5638-5643. doi:10.1073/pnas.0611721104

Raz N, Rodrigue KM, Head D, Kennedy KM, Acker JD. 2004. Differential aging of the medial temporal lobe: a study of a five-year change. Neurology 62: 433-438. doi:10.1212/01.WNL.0000106466.09835.46

Reagh ZM, Yassa MA. 2014. Object and spatial mnemonic interference differentially engage lateral and medial entorhinal cortex in humans. Proc Natl Acad Sci 111: E4264-E4273. doi:10.1073/pnas.1411250111

Reagh ZM, Roberts JM, Ly M, Diprospero N, Murray E, Yassa MA. 2014. Spatial discrimination deficits as a function of mnemonic interference in aged adults with and without memory impairment. Hippocampus 24: 303-314. doi:10.1002/hipo.22224

Reagh ZM, Noche JA, Tustison NJ, Delisle D, Murray EA, Yassa MA. 2018. Functional imbalance of anterolateral entorhinal cortex and hippocampal dentate/CA3 underlies age-related object pattern separation deficits. Neuron 97: 1187-1198.e4.

Rodgers MK, Sindone JA, Moffat SD. 2012. Effects of age on navigation strategy. Neurobiol Aging 33: 326-335. doi:10.1016/j.neurobiolaging .2010.07.021

Rosano C, Guralnik J, Pahor M, Glynn NW, Newman AB, Ibrahim TS, Erickson K, Cohen R, Shaaban CE, MacCloud RL, et al. 2017. Hippocampal response to a 24-month physical activity intervention in sedentary older adults. Am J Geriatr Psychiatry 25: 209-217. doi:10.1016/ j.jagp.2016.11.007

Sahay A, Scobie KN, Hill AS, O'Carroll CM, Kheirbek MA, Burghardt NS, Fenton AA, Dranovsky A, Hen R. 2011. Increasing adult hippocampal 
neurogenesis is sufficient to improve pattern separation. Nature 472: 466-470. doi:10.1038/nature09817

Sorrells SF, Paredes MF, Cebrian-Silla A, Sandoval K, Qi D, Kelley KW, James D, Mayer S, Chang J, Auguste KI, et al. 2018. Human hippocampal neurogenesis drops sharply in children to undetectable levels in adults. Nature 555: $377-381$. doi:10.1038/nature25975

Stark SM, Stark CEL. 2017. Age-related deficits in the mnemonic similarity task for objects and scenes. Behav Brain Res 333: 109-117. doi:10.1016/j .bbr.2017.06.049

Stark SM, Yassa MA, Stark CEL. 2010. Individual differences in spatial pattern separation performance associated with healthy aging in humans. Learn Mem 17: 284-288. doi:10.1101/lm.1768110

Stark SM, Yassa MA, Lacy JW, Stark CEL. 2013. A task to assess behavioral pattern separation (BPS) in humans: data from healthy aging and mild cognitive impairment. Neuropsychologia 51: 2442-2449. doi:10.1016/j .neuropsychologia.2012.12.014

Stark SM, Stevenson R, Wu C, Rutledge S, Stark CEL. 2015. Stability of age-related deficits in the mnemonic similarity task across task variations. Behav Neurosci 129: 257-268. doi:10.1037/bne0000055

Strauss E, Sherman EMS, Spreen O. 2006. A compendium of neuropsychological tests: administration, norms, and commentary, 3rd ed. Oxford University Press, New York, NY.

Suwabe K, Hyodo K, Byun K, Ochi G, Fukuie T, Shimizu T, Kato M, Yassa MA, Soya H. 2017a. Aerobic fitness associates with mnemonic discrimination as a mediator of physical activity effects: evidence for memory flexibility in young adults. Sci Rep 7: 5140. doi:10.1038/ s41598-017-04850-y

Suwabe K, Hyodo K, Byun K, Ochi G, Yassa MA, Soya H. 2017b. Acute moderate exercise improves mnemonic discrimination in young adults. Hippocampus 27: 229-234. doi:10.1002/hipo.22695

Suwabe K, Byun K, Hyodo K, Reagh ZM, Roberts JM, Matsushita A, Saotome K, Ochi G, Fukuie T, Suzuki K, et al. 2018. Rapid stimulation of human dentate gyrus function with acute mild exercise. Proc Natl Acad Sci 115: 10487-10492. doi:10.1073/pnas.1805668115

Tolppanen A-M, Solomon A, Kulmala J, Kåreholt I, Ngandu T, Rusanen M, Laatikainen T, Soininen H, Kivipelto M. 2014. Leisure-time physical activity from mid- to late life, body mass index, and risk of dementia. Alzheimer's Dement 11: 434-443.e6. doi:10.1016/j.jalz.2014.01.008

Tombaugh TN. 2004. Trail making test A and B: normative data stratified by age and education. Arch Clin Neuropsychol 19: 203-214. doi:10.1016/ S0887-6177(03)00039-8 van Praag H, Kempermann G, Gage FH. 1999. Running increases cell proliferation and neurogenesis in the adult mouse dentate gyrus. Nat Neurosci 2: 266-270. doi:10.1038/6368

van Praag H, Shubert T, Zhao C, Gage FH. 2005. Exercise enhances learning and hippocampal neurogenesis in aged mice. J Neurosci 25: 8680-8685. doi:10.1523/JNEUROSCI.1731-05.2005

van Strien NM, Cappaert NLM, Witter MP. 2009. The anatomy of memory: an interactive overview of the parahippocampal-hippocampal network. Nat Rev Neurosci 10: 272-282. doi:10.1038/nrn2614

Varma VR, Chuang YF, Harris GC, Tan EJ, Carlson MC. 2015. Low-intensity daily walking activity is associated with hippocampal volume in older adults. Hippocampus 25: 605-615. doi:10.1002/hipo.22397

Vivar C, Peterson BD, van Praag H. 2016. Running rewires the neuronal network of adult-born dentate granule cells. Neuroimage 131: 29-41. doi:10.1016/j.neuroimage.2015.11.031

Whiteman AS, Young DE, Budson AE, Stern CE, Schon K. 2016. Entorhinal volume, aerobic fitness, and recognition memory in healthy young adults: a voxel-based morphometry study. Neuroimage 126: 229-238. doi:10.1016/j.neuroimage.2015.11.049

Williams ME, Graves LV, DeJesus SY, Holden HM, DeFord NE, Gilbert PE. 2019. Spatial memory ability during middle age may depend on level of spatial similarity. Learn Mem 26: 20-23. doi:10.1101/lm.048280.118

Yassa MA, Muftuler LT, Stark CEL. 2010a. Ultrahigh-resolution microstructural diffusion tensor imaging reveals perforant path degradation in aged humans in vivo. Proc Natl Acad Sci 107: 1268712691. doi:10.1073/pnas.1002113107

Yassa MA, Stark SM, Bakker A, Albert MS, Gallagher M, Stark CEL. $2010 \mathrm{~b}$. High-resolution structural and functional MRI of hippocampal CA3 and dentate gyrus in patients with amnestic mild cognitive impairment. Neuroimage 51: 1242-1252. doi:10.1016/j.neuroimage.2010.03.040

Yassa MA, Lacy JW, Stark SM, Albert MS, Gallagher M, Stark CEL. 2011. Pattern separation deficits associated with increased hippocampal CA3 and dentate gyrus activity in nondemented older adults. Hippocampus 21: 968-979. doi:10.1002/hipo.20808

Received June 25, 2019; accepted in revised form December 10, 2019. 


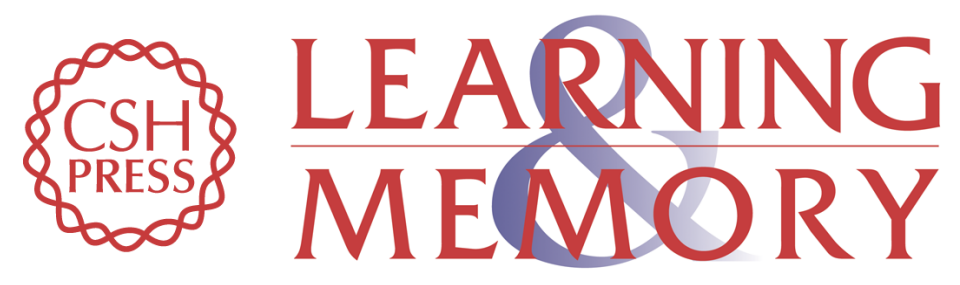

\section{Cardiorespiratory fitness and mnemonic discrimination across the adult lifespan}

Rachel K. Nauer, Karin Schon and Chantal E. Stern

Learn. Mem. 2020, 27:

Access the most recent version at doi:10.1101/Im.049197.118

\section{Supplemental http://learnmem.cshlp.org/content/suppl/2020/01/30/27.3.91.DC1 Material}

References This article cites 74 articles, 13 of which can be accessed free at: http://learnmem.cshlp.org/content/27/3/91.full.html\#ref-list-1

Creative This article is distributed exclusively by Cold Spring Harbor Laboratory Press for the Commons first 12 months after the full-issue publication date (see

License http://learnmem.cshlp.org/site/misc/terms.xhtml). After 12 months, it is available under a Creative Commons License (Attribution-NonCommercial 4.0 International), as described at http://creativecommons.org/licenses/by-nc/4.0/.

Email Alerting Receive free email alerts when new articles cite this article - sign up in the box at the Service top right corner of the article or click here. 